\title{
Speech Recognition by Indexing and Sequencing
}

\author{
BY
}

SIMONE FRANZINI

M.S., University of Illinois at Chicago, 2007

M.S., Politecnico di Milano, Italy, 2006

B.S., Politecnico di Milano, Italy, 2004

\begin{abstract}
DISSERTATION
Submitted in partial fulfillment of the requirements for the degree of Doctor of Philosophy in Computer Science in the Graduate College of the University of Illinois at Chicago, 2012
\end{abstract}

Chicago, Illinois

Defense Committee:

Jezekiel Ben-Arie, Chair and Advisor

Piotr Gmytrasiewicz

Barbara Di Eugenio

Milos Zefran, Electrical and Computer Engineering

Daniel Graupe, Electrical and Computer Engineering 
Copyright by

Simone Franzini 


\section{TABLE OF CONTENTS}

CHAPTER

PAGE

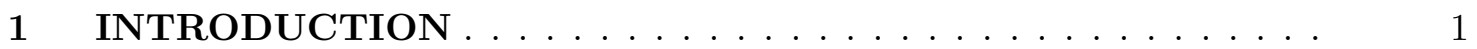

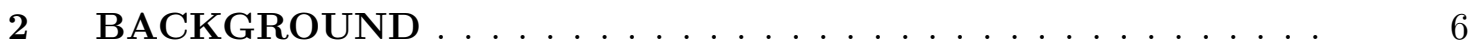

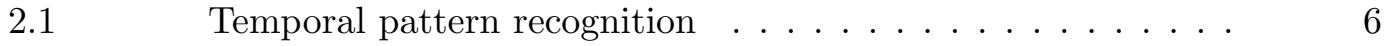

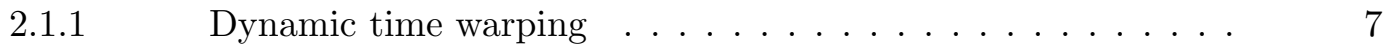

2.1.2 Hidden Markov Models . . . . . . . . . . . . . . . . 9

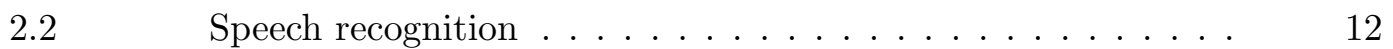

2.2.1 DTW-based speech recognition . . . . . . . . . . 14

2.2.2 HMM-based statistical speech recognition . . . . . . . . . . . . . 14

$2.3 \quad$ Sphinx 4: an HMM-based speech recognizer . . . . . . . . 18

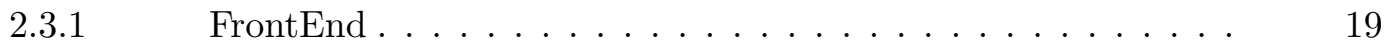

$2.3 .2 \quad$ Linguist $\ldots \ldots \ldots \ldots \ldots \ldots \ldots$

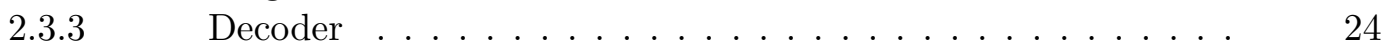

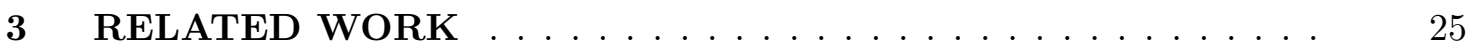

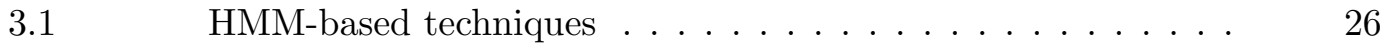

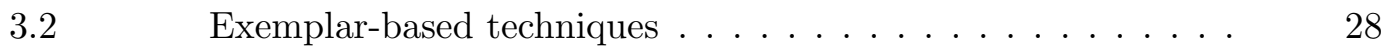

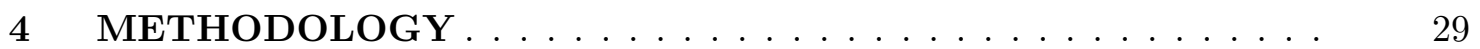

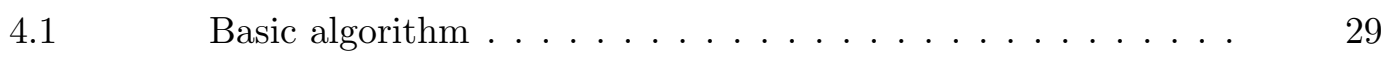

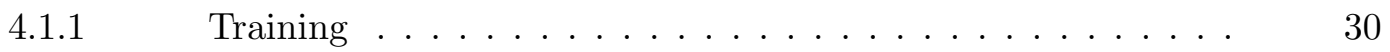

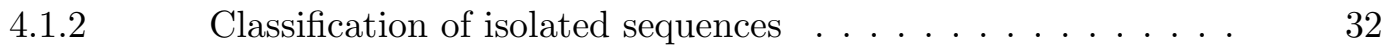

4.1.3 Classification of continuous data streams . . . . . . . . . 38

$4.2 \quad$ Application to speech recognition . . . . . . . . . . . 40

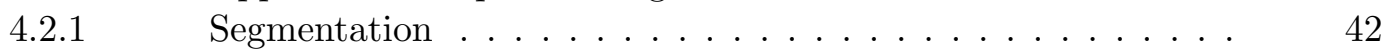

$4.2 .2 \quad$ Dissimilarities . . . . . . . . . . . . . . . . . . . 43

4.2.3 Automatic detection of parts ........... 44

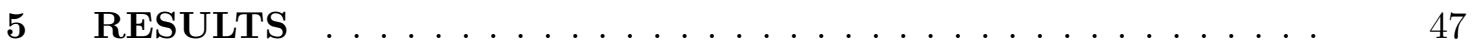

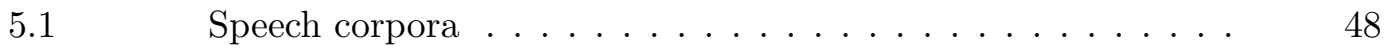

$5.1 .1 \quad$ CSLU Speaker Recognition . . . . . . . . . . . . . 50

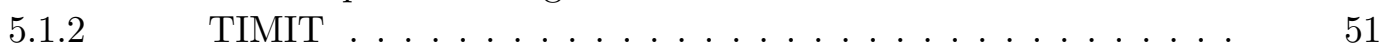

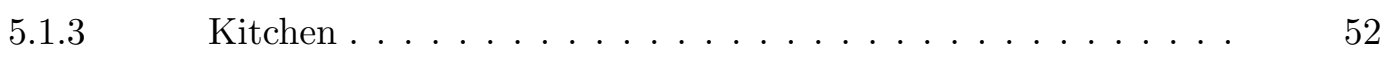

$5.2 \quad$ Isolated-word speech recognition results $\ldots \ldots \ldots \ldots$

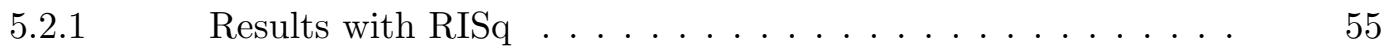

$5.2 .2 \quad$ Results with $\operatorname{Sphinx} \ldots \ldots \ldots \ldots \ldots$

$5.3 \quad$ Continuous speech recognition results . . . . . . . . 59 


\section{TABLE OF CONTENTS (Continued)}

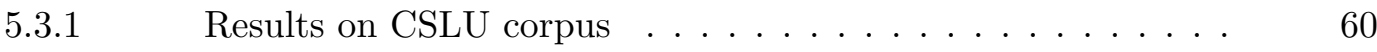

5.3.2 Results on TIMIT corpus . . . . . . . . . . . 62

$5.3 .3 \quad$ Results on Kitchen corpus . . . . . . . . . . . . 64

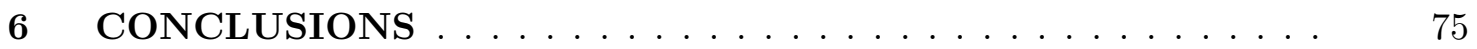

CITED LITERATURE $\ldots \ldots \ldots \ldots \ldots \ldots \ldots$

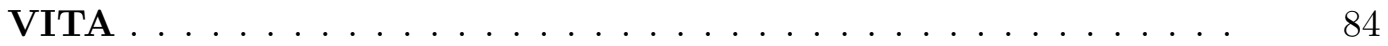




\section{LIST OF TABLES}

\section{TABLE}

PAGE

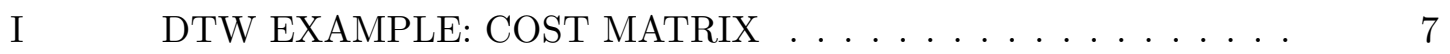

II DTW EXAMPLE: MINIMUM-COST PATH . . . . . . . 8

III WORDS IN THE KITCHEN DICTIONARY AND THEIR RELATIVE FREQUENCIES IN THE ROBOHELPER CORPUS . . . . . 53

IV RESULTS ON CSLU CORPUS: PER-CLASS TPR WHEN FPR=0.1 57

V DETAILED RESULTS ON TIMIT CORPUS WITH RISQ WITH SENTENCE-SPECIFIC DICTIONARY . . . . . . . . . . . 62

VI DETAILED RESULTS ON TIMIT CORPUS WITH SPHINX WITH SENTENCE-SPECIFIC DICTIONARY . . . . . . . . . . . .

VII DETAILED RESULTS ON KITCHEN CORPUS WITH RISQ WITH SENTENCE-SPECIFIC DICTIONARY . . . . . . . . . . . . .

VIII DETAILED RESULTS ON KITCHEN CORPUS WITH SPHINX WITH SENTENCE-SPECIFIC DICTIONARY . . . . . . . . . 66

IX SUMMARY OF RESULTS WITH SENTENCE-SPECIFIC DICTIO-

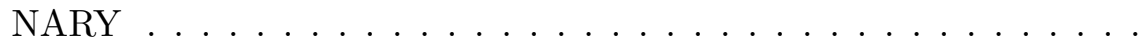

X DETAILED RESULTS ON KITCHEN CORPUS WITH RISQ WITH FULL DICTIONARY . . . . . . . . . . . . . . . . 71

XI DETAILED RESULTS ON KITCHEN CORPUS WITH SPHINX WITH FULL DICTIONARY . . . . . . . . . . . . . 72

XII SUMMARY OF RESULTS WITH FULL DICTIONARY . . . . . 73

XIII DETAILED RESULTS ON KITCHEN CORPUS WITH GOOGLE SPEECH RECOGNIZER . . . . . . . . . . . . . . . . . 


\section{LIST OF FIGURES}

FIGURE

PAGE

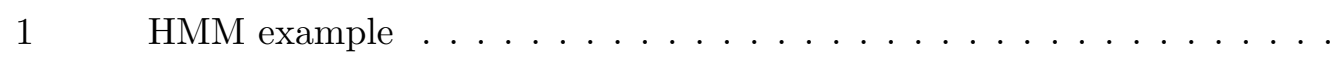

$2 \quad$ Architecture of $\operatorname{Sphinx} 4 \ldots \ldots \ldots$

3 Extraction of MFCCs from speech data $\ldots \ldots \ldots \ldots$

4 Example of $2 \mathrm{D}$-tree $\ldots \ldots \ldots \ldots$

5 Example of range search on a 2 D-tree $\ldots \ldots \ldots \ldots$

$6 \quad$ Sequencing example $\ldots \ldots \ldots \ldots \ldots$

$7 \quad$ Pseudocode of indexing and sequencing $\ldots \ldots \ldots$

$8 \quad$ Extraction of features from speech data for RISq . . . . . . . . . . . 40

9 Autocorrelation on the word "glass" $\ldots \ldots \ldots$

10 Values of the convolution of the autocorrelation of the word "glass" with masks of different sizes $\ldots$

11 Results on CSLU corpus: average ROCs . . . . . . . . . . . . .

12 Example of RISq applied to continuous speech . . . . . . . . . .

13 Recognition of a sentence with RISq with sentence-specific dictionary .

14 Recognition of a sentence with RISq with full dictionary . . . . . . . . . 


\section{LIST OF ABBREVIATIONS}

ANN

ASR

CDHMM

CRF

CSLU

DTW

EM

HHMM

HMM

HTK

LHMM

MEMM

MFCC

MFFB

MRF

NIST

PLP
Artificial Neural Network

Automatic Speech Recognition

Continuous Density Hidden Markov Model

Conditional Random Field

Center for Spoken Language Understanding

Dynamic Time Warping

Expectation-Maximization

Hierarchical Hidden Markov Model

Hidden Markov Model

HMM ToolKit

Layered Hidden Markov Model

Maximum Entropy Markov Model

Mel-Frequency Cepstral Coefficient

Mel-Frequency Filter Bank

Markov Random Field

National Institute of Standards and Technology

Perceptual Linear Prediction

vii 


\section{LIST OF ABBREVIATIONS (Continued)}

$\begin{array}{ll}\text { RISq } & \text { Recognition by Indexing and Sequencing } \\ \text { ROC } & \text { Receiver Operating Characteristic } \\ \text { RTF } & \text { Real Time Factor } \\ \text { SVM } & \text { Support Vector Machine } \\ \text { WER } & \text { Word Error Rate } \\ \text { WSJ } & \text { Wall Street Journal }\end{array}$




\section{SUMMARY}

Recognition by Indexing and Sequencing (RISq) is a general-purpose method for classification of temporal vector sequences, that was previously applied to human activity recognition. We have developed an advanced version of RISq and have adapted it to continuous speech recognition, a challenging task most commonly solved today with Hidden Markov Models (HMMs).

Despite being very popular, HMMs suffer from some intrinsic problems, such as the need for a very large amount of training data in order to build a model for each class to be recognized. As opposed to HMMs, RISq consists of a non-parametric algorithm that can be trained with as little as one exemplar for each class. This makes RISq more suitable for tasks where only a limited amount of training data is available or where there is significant intra-class variation.

Moreover, from a practical standpoint even the most powerful HMM-based speech recognizers have achieved only modest performance improvements over the last two decades. This lead to renovated interest in non-parametric exemplar-based techniques such as RISq. Because of its ease of training, RISq can adapt extremely fast to a new speaker. In fact, it is possible to train RISq for speakers of different languages or with significantly different accents.

The basic RISq methodology consists of a two-step classification algorithm: first the training samples closest to each input sample are identified and weighted with a parallel algorithm (indexing). Then a maximum weighted bipartite graph matching is found between the input sequence and a training sequence, respecting an additional temporal constraint (sequencing). 


\section{SUMMARY (Continued)}

The adaptation of RISq to speech recognition lead to several general-purpose improvements of the basic algorithm. The basic methodology was extended to use dissimilarity measures as well as similarity. In order to account for variability in the input data, several exemplars of each class to be recognized are used for training. Moreover, we have devised a novel algorithm to automatically identify parts of the training sequences (i.e. words). Finally, we have developed a segmentation approach to apply RISq to continuous speech, as well as a post-processing technique to identify the timing of detected words.

Experimental evaluation was conducted on the standard TIMIT speech database, as well as on a small corpus recorded in our lab. The performance of RISq was compared with that of Sphinx, a state-of-the-art HMM-based system. Results show how RISq performs at least as well as Sphinx in most of our tests, outperforming Sphinx in the recognition of a non-native American English speaker. 


\section{CHAPTER 1}

\section{INTRODUCTION}

Temporal pattern recognition is an important task with applications in many different areas. Patterns can be identified in different kinds of time-varying signals, such as speech, gestures and human activities. The problem can be stated as follows: given some example sequences for each of $\mathbf{C}$ classes $\mathbf{Y}_{\mathbf{c}}$, classify an input sequence $\mathbf{Y}_{\mathbf{t}}$ as belonging to the most similar class. There are two main classes of algorithms that can solve this problem. Parametric techniques first estimate a model for each class from the training data, then estimate the probability that the input signal belongs to each of those classes. Non-parametric techniques perform recognition directly by comparing the input with the training data.

One of the most interesting and most challenging areas of application is speech recognition, which consists in converting speech to text. Since its inception in the 60s, speech recognition proved to be a much harder task than initially thought and, despite a consistent progress in the field, it still constitutes a widely open problem. In spite of many efforts, most of the continuousspeech recognizers seem to have hit an accuracy plateau of about $80 \%$ in the early 2000 s with no significant improvements in the last decade (1). While this might seem a high recognition rate, it is still not enough for natural interaction. Even when using simple automated responding systems, many users still experience frustrating performance. If automated speech recognition is to become the primary input mode for computers, a higher and more consistent performance needs to be achieved. 
The two most popular methods that have been applied to temporal pattern recognition and speech recognition are Dynamic Time Warping (DTW) and Hidden Markov Models (HMMs).

Dynamic Time Warping (DTW) (2) is a general-purpose non-parametric technique developed in the 70s to align temporal or spatial sequences. While DTW was the first method applied to speech recognition, it was eventually replaced by Hidden Markov Models (HMMs).

Hidden Markov Models (HMMs) (3) are a complex parametric technique for classification of temporal sequences. Despite being invented in the 60s, HMMs only began to gain popularity in the 80 s, when they started to be successfully applied to speech recognition (4). The inherent complexity of HMMs is due to the fact that it is necessary to build a model for each class and to estimate a very large number of parameters for each model. This in turn leads to a long and complicated Expectation-Maximization (EM) training algorithm (5) that requires a large amount of data. Current HMM-based Automatic Speech Recognition (ASR) systems have become very complex, while still failing to deliver a high recognition rate.

There are two important limitations to this approach, both due to the need of building statistical models based on a large amount of data. First, the models are built based on speech from native speakers of a language, so users who are non-native or with heavy accents usually experience poor performance. Adaptation to a new user is possible, but it usually leads to very modest improvements if the user is too far from the population used for training. Second, the approach relies on very large annotated speech corpora for each language, which are not available for many languages. These two problems have been identified as a substantial challenge for the next generation of ASR systems $(6 ; 7)$. 
There is some agreement in the scientific community that a substantial paradigm shift in speech recognition might help to solve some of the problems that are limiting the performance of HMM-based systems. This lead in recent years to an increasing interest in exemplar-based (also called template-based) techniques for speech recognition $(8 ; 9)$. While eliminating the need to build models and estimate many parameters, these two approaches are still based on a typical HMM framework.

Most of the current HMM-based systems are built for speaker-independent speech recognition. The reason for this is that building accurate acoustic models is an extremely time consuming task. Other than requiring a very large amount data, it also needs very careful tuning of many parameters, which cannot be entirely automated. Therefore, standard acoustic models are distributed to be used in conjunction with these systems. However, being based on averages, they can only work well for speakers with similar characteristics as those used in the corpus. Most of these speakers are usually adult native speakers of a certain language. Foreigners, people with heavy accents, children and elderly will experience lowered recognition rates from such a system. On the other hand, completely training acoustic models to a specific speaker, i.e. performing speaker-dependent speech recognition, is not feasible for HMM-based systems. This would require a very large amount of data from a speaker, as well as expert manual tuning of parameters. A partial solution is to adapt standard acoustic models to a specific speaker, however this might not improve the recognition by a large percentage. 
In this work, we propose the application of Recognition by Indexing and Sequencing (RISq) $(10 ; 11)$ to speech recognition $(12 ; 13)$. RISq is a non-parametric technique that applies a classical pattern recognition approach designed for single vectors and modifies it for matching of vector sequences. RISq presents definite advantages with respect to both DTW and HMMs. RISq uses a simple training procedure and can achieve robust recognition even when training is performed with only one example sequence from each class. It is also possible to train RISq with many independent example sequences for the same class. This allows the method to efficiently address intra-class variability. Also, classification can be performed using only a few samples from the input sequence. Additionally, RISq is more robust than HMMs to noise and missing information and recognition is partially parallel whereas with HMMs or DTW it is completely serial. These advantages are particularly critical for applications where only a few training data examples are available and significant differences are present among examples of the same class.

Because of these advantages, RISq makes speaker-dependent speech recognition not only possible, but also practical. This could lead to significant improvements for many speakers and situations, such as a multi-modal effective communication interface for the elderly (14) that we are currently developing. In this project, RISq is used to perform speech recognition of elderly people, who may have distorted or slowed down speech and where fast adaptation to new users is essential. Standard acoustic models developed from adult speakers will not work properly for the elderly, because physical changes occurring with aging significantly affect speech (15). Therefore, training on an elderly-specific dataset or, even better, on a particular speaker's voice, needs to occur in order to achieve a sufficient level of performance. 
In principle, RISq can recognize any kind of spatial or temporal sequence, such as human activities, to which it was already successfully applied (10). The original formulation of RISq is described in (11), which also suggests its possible application to speech. The major contributions of this work are improvements to the basic methodology, such as the use of a more efficient data structure and a sequencing algorithm with a lower computational complexity, and the novel adaptation of RISq to both isolated-word and continuous speech recognition. The recognition of continuous speech is particularly challenging and we had to develop many improvements of the basic methodology to address the complexity of this task. Moreover, most of the novel ideas that we developed for speech recognition are completely general and can be applied to other kind of signals as well. In order to validate the experimental results, the performance of RISq is compared to that of Sphinx, a state-of-the-art HMM-based speech recognizer, on two standard speech databases, as well as a small corpus that we recorded.

The remainder of this thesis is organized as follows. In Chapter 2 we explain how DTW and HMMs work, both as general temporal pattern recognition methods and as applied to speech recognition. In Chapter 3 we explore state-of-the-art literature about speech recognition. In Chapter 4 we describe the methodology of our approach and its application to speech recognition. In Chapter 5 we provide results for the application of RISq to several speech corpora and a comparison with state-of-the-art HMM-based speech recognizers. Finally in Chapter 6 we summarize the content of this work and provide guidelines for future work. 


\section{CHAPTER 2}

\section{BACKGROUND}

In order to understand the innovative concepts proposed in RISq and how it improves on the state-of-the-art, it is first necessary to understand the basic ideas underlying Dynamic Time Warping and Hidden Markov Models. In this chapter we describe the problems of temporal pattern recognition and speech recognition. We also explain how DTW and HMM have been used to solve these problems. In Section 2.1 we show a simple example of how DTW and HMM work. In Section 2.2 we discuss how these techniques are applied to speech recognition. Finally, in Section 2.3 we analyze Sphinx, a state-of-the-art HMM-based speech recognition system. By analyzing the complex architecture of Sphinx, it is possible to appreciate the much simpler structure of RISq.

\subsection{Temporal pattern recognition}

Temporal pattern recognition consists in the identification of patterns in time-varying signals. This can be performed on isolated events or on a continuous data stream. When applied to speech, this translates to isolated-word speech recognition and continuous speech recognition. In this section we describe two general-purpose methods that can be applied to temporal pattern recognition: Dynamic Time Warping and Hidden Markov Models. 


\subsubsection{Dynamic time warping}

Dynamic Time Warping (DTW) (2) is a non-parametric technique that matches temporal or spatial sequences. In its original formulation, DTW can only be applied to two monodimensional vectors: first a cost matrix is built to measure the distance from each element of one sequence to each element of the other one; then a minimum-cost path is found with a dynamic programming algorithm. In Table I we see the cost matrix between the sequences $x=[0,1,1,2,3,3,2]$ and $y=[1,1,2,3,1]$, computed according to the distance function $(x-y)^{2}$.

\begin{tabular}{|l||l|l|l|l|l|l|l|}
\hline & 0 & 1 & 1 & 2 & 3 & 3 & 2 \\
\hline \hline 1 & 1 & 0 & 0 & 1 & 4 & 4 & 1 \\
\hline 1 & 1 & 0 & 0 & 1 & 4 & 4 & 1 \\
\hline 2 & 4 & 1 & 1 & 0 & 1 & 1 & 0 \\
\hline 3 & 9 & 4 & 4 & 1 & 0 & 0 & 1 \\
\hline 1 & 1 & 0 & 0 & 1 & 4 & 4 & 1 \\
\hline
\end{tabular}

TABLE I

DTW EXAMPLE: COST MATRIX

In Table II we see the minimum-cost matrix, computed according to the following dynamic programming formula:

$$
\operatorname{DTW}(i, j)=\operatorname{cost}(i, j)+\min (\operatorname{DTW}(i, j-1), \operatorname{DTW}(i-1, j-1), \operatorname{DTW}(i-1, j))
$$


The $(0,0)$ element has been initialized to 0 and all of the other 0 -th elements have been initialized to infinity. The minimum cost to align the sequences is 2 , the value found in the lower right corner of the table. A minimum-cost path is marked in bold. Note how in this case there is no unique minimum-cost path, as the first 1 from the top sequence can be aligned either with the first or the second 1 in the left sequence with the same cost.

\begin{tabular}{|c||c|c|c|c|c|c|c|c|}
\hline & & 0 & 1 & 1 & 2 & 3 & 3 & 2 \\
\hline & 0 & $\infty$ & $\infty$ & $\infty$ & $\infty$ & $\infty$ & $\infty$ & $\infty$ \\
\hline 1 & $\infty$ & $\mathbf{1}$ & 1 & 1 & 2 & 6 & 10 & 11 \\
\hline 1 & $\infty$ & 2 & $\mathbf{1}$ & $\mathbf{1}$ & 2 & 6 & 10 & 11 \\
\hline 2 & $\infty$ & 6 & 2 & 2 & $\mathbf{1}$ & 2 & 3 & 3 \\
\hline 3 & $\infty$ & 15 & 6 & 6 & 2 & $\mathbf{1}$ & $\mathbf{1}$ & 2 \\
\hline 1 & $\infty$ & 16 & 6 & 6 & 3 & 5 & 5 & $\mathbf{2}$ \\
\hline
\end{tabular}

TABLE II

\section{DTW EXAMPLE: MINIMUM-COST PATH}

Only recently DTW has been extended to multi-dimensional vectors (16) and multiple sequences (17). A major disadvantage of dynamic time warping is that a continuous path must be built to match each sample in one sequence to each sample in the other sequence. This requirement does not allow for matching of sequences that have been interrupted by small gaps of interference as it frequently occurs in practice. Also DTW requires the knowledge of the 
endpoints of the sequences before matching can be attempted, which makes its application to continuous data streams very difficult and heavily dependent on the segmentation algorithm.

\subsubsection{Hidden Markov Models}

Hidden Markov Models (HMMs) (3) are a parametric method based on a maximum likelihood approach and have been used successfully to perform pattern recognition tasks, where sequences that unfold in time are involved. A Markov model is used to model a Markov process, one that has the Markov property, i.e. where the conditional probability distribution of future states of the process, given the present state and the past states, depends only upon the present state. The simplest Markov model is a Markov chain, which models the state of a system with a time-varying random variable. Differently than a Markov chain, a HMM assumes that the state of the variable is hidden and can only be inferred through observations. A HMM can be seen as a simple kind of dynamic Bayesian network or as a generalization of a mixture model.

A Markov model is built up of a set of states and a matrix of transition probabilities from one state to another. In a HMM, the internal states are hidden and can only be inferred through observations, i.e. outputs of each state, that can be modeled either as a discrete or continuos probability function. HMMs using continuous probability functions to model observations are sometimes called Continuous Density HMMs (CDHMMs).

Here is a concrete example. Consider two friends, Alice and Bob, who live far apart from each other and who talk together daily over the telephone about what they did that day. Bob is only interested in three activities: walking in the park, shopping, and cleaning his apartment. The choice of what to do is determined exclusively by the weather on a given day. Alice has no 
definite information about the weather where Bob lives, but she knows general trends. Based on what Bob tells her he did each day, Alice tries to guess what the weather must have been like. Alice believes that the weather operates as a discrete Markov chain. There are two states, "Rainy" and "Sunny", but she cannot observe them directly, that is, they are hidden from her. On each day, there is a certain chance that Bob will perform one of the following activities, depending on the weather: "walk", "shop", or "clean". Since Bob tells Alice about his activities, those are the observations. The system can be modeled by the HMM in Figure 1.

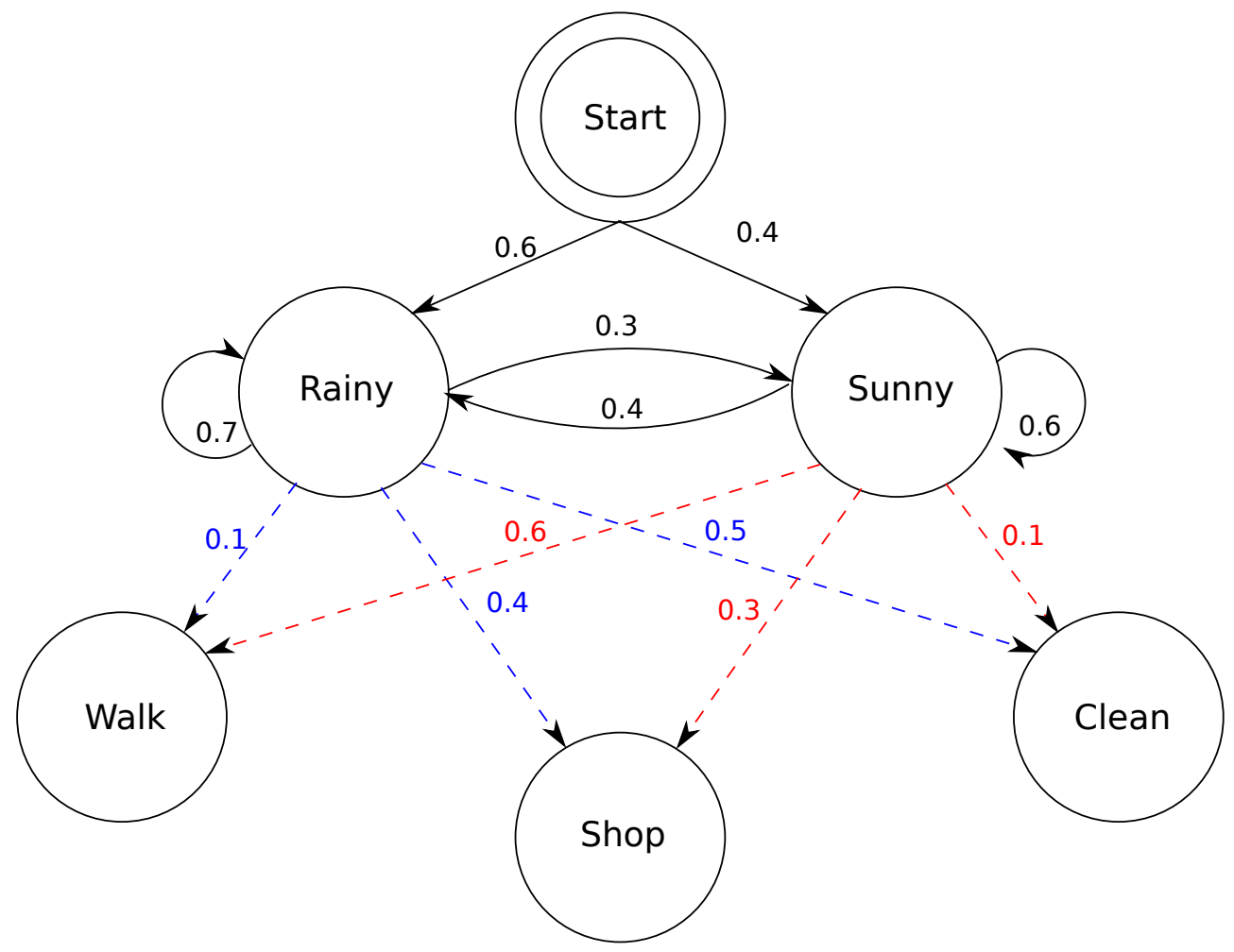

Figure 1. HMM example 
There are three main problems involved in the use of HMMs (4):

1. The evaluation problem: given an HMM, with known transition probabilities and output probability function, determine the likelihood that a sequence of visible states was generated by that model. This problem is efficiently solved by either the forward or the backward algorithm.

2. The decoding problem: given an HMM, with known transition probabilities and output probability function, determine the most likely sequence of internal states that generated a sequence of visible states. This problem is efficiently solved by the Viterbi algorithm.

3. The learning problem: given the structure of an HMM, that is the number of states and the type of output probability function, determine the transition probabilities and parameters of the function, given a set of sequences of observed states. This is basically the training procedure, which adapts an HMM to a certain kind of signals to be recognized. This problem is solved by the Baum-Welch algorithm (18).

When building a multi-class classifier, it is necessary to train an HMM for each model to be recognized, which is problem 3 above. An input sequence is then tested against all the models, and it is classified according to the model which yields the highest likelihood. In other words, that model is the one which best explains the sequence, which is problem 1 above.

The learning problem is by far the most complicated: the Baum-Welch algorithm is a generalized Expectation-Maximization (EM) algorithm, which requires a large number of examples for proper estimation of the parameters. 
Training is further complicated by the fact that there is no general algorithm to determine the structure of an HMM, that is the number of states and connections between them, which is more suitable to recognize a certain kind of data.

\section{$2.2 \quad$ Speech recognition}

Speech recognition consists in converting speech to text. It can be either speaker-dependent, a simpler task, or speaker-independent, a more complex task. The reason why the former is easier is that there is only intra-speaker variability. In the second case, the added inter-speaker variability requires some approach to eliminate the differences among speakers while preserving the common characteristics. This task is the opposite of speaker recognition, in which the differences among speakers are accentuated and the common characteristics eliminated.

Speech recognition has applications in a large number of fields and it is particularly useful because it allows to develop a more natural way of communication with a machine, allowing to at least partially substitute input devices such as keyboard or similar hardware. An application can be categorized as small, medium or large vocabulary, depending on the number of different words that it needs to recognize. The borders between these classes are not precisely defined, however a vocabulary is usually considered small when it comprises no more than about 100 words, medium up to a few thousands words and large thereafter. For example, simple applications recognizing voice commands are likely to need only a small vocabulary, whereas more complex user interfaces may need a medium-size vocabulary and dictation usually needs a large vocabulary. The difficulty of the speech recognition task increases with the size of the vocabulary. It also increases when going from isolated-word to connected-word (i.e. continuous) 
speech recognition. This increase in complexity is due to the fact that most of the techniques need to know where each word starts and ends, a task referred to as audio segmentation. This is a very hard problem in itself and still constitutes an interesting research area.

Since the introduction of commercial speech recognition systems in the 70 s, just a few successful systems have been developed so far, most of them being the result of decades of experience from large companies, such as IBM, Apple, Microsoft and Philips. The diffusion of speech recognition technology has been much slower than expected, mainly due to two reasons: the required performance and the time needed for training. To be of any practical use, speech recognition systems must achieve very high recognition rates which have not yet been reached consistently by any current system. Moreover, most of the systems need to be fine tuned to the user's voice, often requiring repeating the same sentences of words for several times, a long and tedious process that also discourages the user. Even after this is done, some users may still experience a much lower recognition rate than advertised. This is usually due to non-ideal conditions, such as background noise or a speaker with an accent that does not appear in the large amount of data used to train the system. In fact, most of the systems are trained with a speech corpus that is standardized for a particular language, for example American English. In this case, the corpus is usually balanced among different American accents. However, a non-native American speaker is likely to have issues with such a system. In the following, we will discuss how DTW and HMMs have been applied to speech recognition. 


\subsubsection{DTW-based speech recognition}

Dynamic Time Warping is a dynamic programming general-purpose technique for sequence alignment; it measures the similarity between two temporal or spatial sequences, by non-linearly warping one into the other. It allows to identify common parts of sequences even when they are initiated at different times or performed at different speeds, i.e. it is basically invariant to time-shift and scale.

The use of DTW for isolated word recognition was pioneered in the 70s in work by Sakoe and Chiba (2), Velichko and Zagoruyko (19), Myers, Rabiner and Rosenberg (20). Despite showing initially promising results, DTW proved unable to handle the inherent complexity of speech, variations between speakers and continuous speech recognition. Particularly limiting factors consisted in working directly with the speech signal, instead of using features extracted from it, and in the need to identify the beginning and the end of the test sequence before a matching could be attempted. Because of these issues, DTW was progressively abandoned through the 80s in favor of the more successful and more complex HMM-based statistical approach.

\subsubsection{HMM-based statistical speech recognition}

In this section, we will first define a generic statistical framework for speech recognition, as described in (21), and then see how HMMs can fit into this scenario.

Let us denote with $\mathbf{A}$ the acoustic data that need to be recognized; we will assume that it is composed of a sequence of symbols from an alphabet $\mathscr{A}$ :

$$
\mathbf{A}=a_{1}, a_{2}, \ldots, a_{m} \quad a_{i} \in \mathscr{A}
$$


Let us denote with $\mathbf{W}$ a string of $\boldsymbol{n}$ words taken from an alphabet $\mathscr{V}$ :

$$
\mathbf{W}=w_{1}, w_{2}, \ldots, w_{n} \quad w_{i} \in \mathscr{V}
$$

The acoustic data will be recognized as the most likely string of words given the acoustic data:

$$
\widehat{\mathbf{W}}=\underset{\mathbf{W}}{\operatorname{argmax}} \mathrm{P}(\mathbf{W} \mid \mathbf{A})
$$

We can rewrite the right-hand side using Bayes' theorem:

$$
\mathrm{P}(\mathbf{W} \mid \mathbf{A})=\frac{\mathrm{P}(\mathbf{A} \mid \mathbf{W}) \mathrm{P}(\mathbf{W})}{\mathrm{P}(\mathbf{A})}
$$

where $P(\mathbf{W})$ is the prior probability of pronouncing the string of words $\mathbf{W}, \mathbf{P}(\mathbf{A} \mid \mathbf{W})$ is the probability of observing the acoustic data $\mathbf{A}$ when $\mathbf{W}$ is pronounced, and $\mathrm{P}(\mathbf{A})$ is the prior probability of hearing the acoustic data. This can be rewritten as:

$$
\mathrm{P}(\mathbf{A})=\sum_{\mathbf{W}^{\prime}} \mathrm{P}\left(\mathbf{A} \mid \mathbf{W}^{\prime}\right) \mathrm{P}\left(\mathbf{W}^{\prime}\right)
$$

But since $\mathbf{A}$ is fixed while maximizing $\mathrm{P}(\mathbf{W} \mid \mathbf{A})$, we can rewrite:

$$
\widehat{\mathbf{W}}=\underset{\mathbf{W}}{\operatorname{argmax}} \mathrm{P}(\mathbf{A} \mid \mathbf{W}) \mathrm{P}(\mathbf{W})
$$

This formula implicitly specifies the components of a speech recognizer. 
First of all, we need a front end to convert the raw speech waveform into the acoustic data A. This is necessary because similar waveforms do not always correspond to sounds perceived as similar. We will describe this signal processing in detail in Section 4.2.

We then need a statistical acoustic model to compute $\mathrm{P}(\mathbf{A} \mid \mathbf{W})$; this model takes into account how the speaker pronounces the words of $\mathbf{W}$ and the characteristics of the components of the communication channel, such as the ambient and the microphone. Hidden Markov Models are generally used for this task.

The prior probability $\mathrm{P}(\mathbf{W})$ can be written as:

$$
P(\mathbf{W})=\prod_{i=1}^{n} P\left(w_{i} \mid w_{1}, \ldots, w_{i}-1\right)
$$

However, it is computationally infeasible (other than being unrealistic) to let the probability of a word depending on the entire sequence of words that precede it. Therefore, this is approximated by assuming that the probability of a word only depends on the probability of the previous n-1 words:

$$
P(\mathbf{W})=\prod_{i=1}^{n} P\left(w_{i} \mid w_{i-(n-1)}, \ldots, w_{i}-1\right)
$$

This approximation is referred to as an n-gram language model, where $\mathrm{n}$ is usually 2 or 3 ; increasing it to a larger value becomes computationally prohibitive, while yielding only a small increase in the recognition rate. These probabilities can be estimated from a word count on a given corpus, which must be very large for them to be accurate. 
Finally, we need to perform a search over all of the possible strings $\mathbf{W}$ in order to find the maximizing one. This is also a computationally infeasible task, which must be performed using some knowledge that prunes most of the possibilities. HMMs can be used in this framework as the acoustic model, in which case this search is performed through either the Viterbi algorithm (a trellis search) or a graph search.

Inside this framework, HMMs proved more successful than DTW in many respects, included the ability of better overcoming differences between speakers through modeling and of handling continuous speech recognition, at the expense of a much increased complexity. In his exhaustive tutorial on HMMs and their use for speech recognition, Rabiner (4) highlights several implementation issues for HMMs, especially regarding the large amount of data needed for training, the training procedure itself and the choice of a proper model.

Being a parametric technique, HMMs also require to maintain a very large number of parameters. For example, in Sphinx (22), a state-of-the-art speech recognizer based on HMMs, the acoustic model for a word is composed of smaller models of the phonemes in the word. Phonemes in turn are composed of three states, an on-set, a middle, and an end, and each of these states is modeled with a mixture of multi-dimensional Gaussians. When D dimensions (i.e. features) and $G$ Gaussians are used, we need to maintain $O\left(D^{2} \times G\right)$ parameters for each state if full covariance matrices are used or at least $\mathrm{O}(\mathrm{D} \times \mathrm{G})$ when only elements on the diagonal are retained. In Sphinx, D = 39 and G varies between 8 and 32, which represent typical values for this kind of system. A complex and data-intensive training procedure is needed to reliably estimate these parameters. 


\subsection{Sphinx 4: an HMM-based speech recognizer}

Sphinx 4 is the most recent version of an HMM-based speech recognizer developed by Carnegie Mellon University, a project that started more than 20 years ago. Sphinx 4 is currently considered a state-of-the-art system. Therefore, it is important to analyze its architecture in some detail, in order to understand how such a system works and the complexity underlying this approach. Most of the information in this section is derived from (22) and (23). The most significant difference of Sphinx 4, with respect to the previous versions, is that it has been completely rewritten in Java using a new modularized, configurable and pluggable architecture, shown in Figure 2.

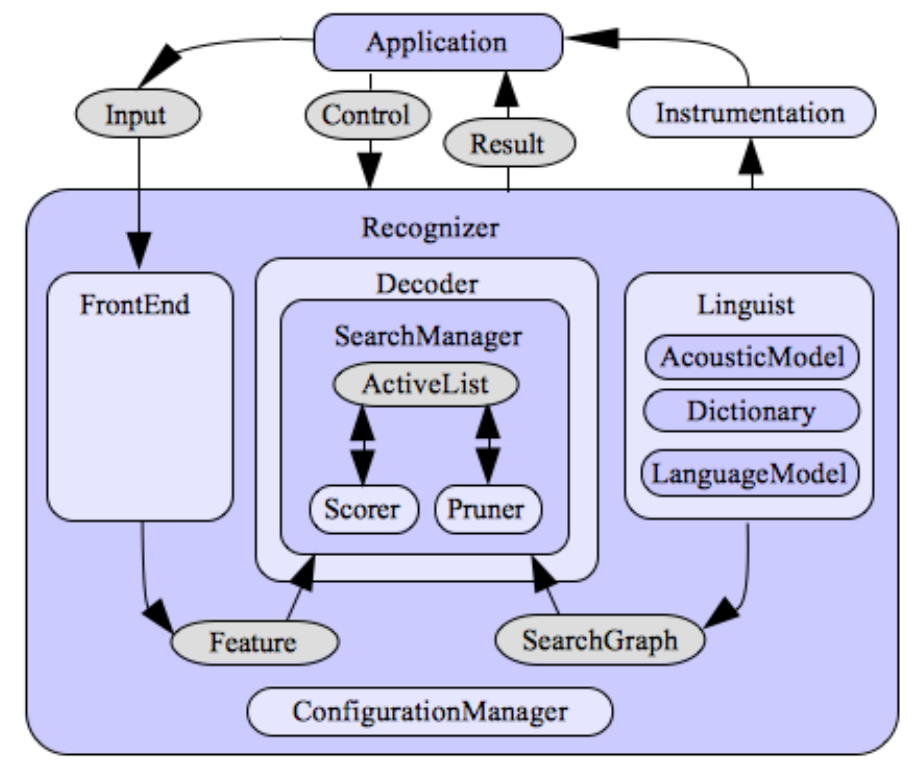

Figure 2. Architecture of Sphinx 4 
As can be seen from Figure 2, the architecture comprises several components. At the core there is a Recognizer, which includes the FrontEnd, the Decoder and the Linguist. All of these components correspond to parts of an HMM-based speech recognizer, as described in Section 2.2.2. The FrontEnd elaborates the raw audio data and extracts informative features that can be used for training and classification. The Linguist creates a search graph for the decoder, using a large amount of domain knowledge in order to reduce the size of the search space. This knowledge comprises the acoustic models, the dictionary and the language model. The Decoder is at the core of the architecture: its SearchManager searches the graph provided by the Linguist, using the features from the input sequence provided by the FrontEnd, in order to decode the speech signal. We will now analyze each of these components in more detail.

\subsubsection{FrontEnd}

The FrontEnd computes features from the audio data, performing several steps of signal processing. It uses chains of generic data processors that communicate with each other using a pull architecture. Both live and batch modes are possible: in live mode data will be fetched from a microphone, in batch mode from previously recorded audio files.

Mel-Frequency Cepstral Coefficients (MFCCs) are the features used by default, but other ones are also supported, such as Perceptual Linear Prediction (PLP) coefficients. MFCCs were first proposed by Davis and Mermelstein (24), who showed that they yielded a higher recognition rate with respect to other common features, such as linear prediction spectrum, linear prediction cepstrum and linear frequency cepstrum.

A cepstrum is obtained by taking the Fourier transform of the spectrum of a signal, as if it were 
a signal itself; its name was derived by inverting the first four letters of the word "spectrum". The cepstrum provides information about the rate of change in the spectrum bands. There are different kinds of cepstra: the power cepstrum and the complex cepstrum, formed by a real cepstrum and a phase cepstrum. Therefore, the exact sequence of operations performed to obtain the cepstrum differs slightly depending on its kind.

For speech recognition, the phase proved to be of minor importance for classification, so the real spectrum is commonly used. Moreover, the spectrum is often first transformed to the non-linear mel-frequency scale, similar to the non-linear function in the human auditory system.

The audio signal processing sequence used in the sample applications comprises preemphasis, windowing with a raised cosine transform, computation of MFCCs, cepstral mean normalization and computation of the first and second order derivatives of the normalized MFCCs. Since 13 MFCCs coefficients are retained, this amounts to using a total of 39 features.

The full procedure with specific parameters for an $8 \mathrm{KHz}$ signal is shown in Figure 3.

The FrontEnd also supports a few more signal processing techniques, such as an endpointer, and can be easily plugged with user-defined data processors.

\subsection{2 $\quad \underline{\text { Linguist }}$}

The task of the Linguist is to create a search graph for the decoder. Depending on the size of the vocabulary, Sphinx provides different implementations of the Linguist in order to optimally achieve this task. The Linguist is composed of three main components: the acoustic models, the dictionary and the language model. 


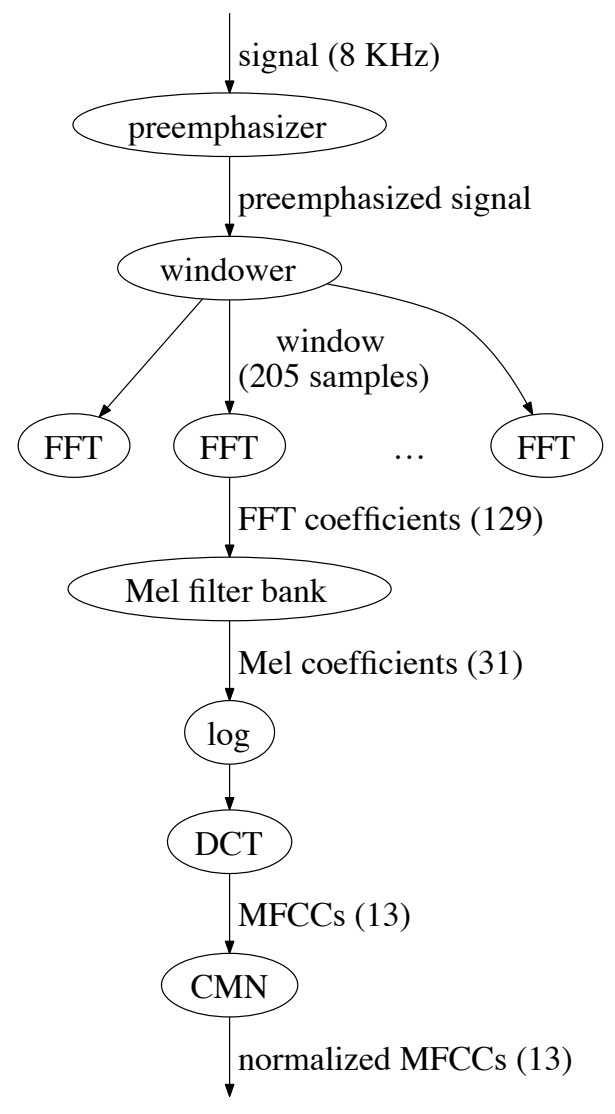

Figure 3. Extraction of MFCCs from speech data 
A language model is necessary when dealing with continuous-word speech recognition in order to reduce its exponential complexity, as explained in Section 2.2.2. Sphinx 4 supports two main kinds of language models: grammars, that work well for small dictionaries, and n-gram models, necessary for larger dictionaries. Generating a good trigram model for a large dictionary is a challenging task itself, since it needs a very large corpus in order to properly estimate the probabilities. For the large trigram model provided with Sphinx 4, this has been done with the CMU-Cambridge Statistical Language Modeling Toolkit. In practice, the probability $\mathrm{P}(\mathbf{W})$ computed according to the language model, is raised to some exponent $\alpha$. Even if this does not have any proper statistical motivation, it has been proven to be critical to achieve a high recognition rate. Optimal values of $\alpha$ used in Sphinx are between 6 and 11 .

A dictionary is necessary to map each word that needs to be recognized to its pronunciation. A word is broken into a sequence of sub-word units, according to the acoustic models. Syllables were used in the past as sub-word units, but phones are now more commonly used. A phone is a definite sound that can be produced by the human vocal tract; there are about only 50 phones and they are independent from the particular language spoken. A phoneme is a basic sound unit that is perceived by native speakers of a particular language as having a specific acoustic role. Several phones can map to the same phoneme for speakers of a language (allophones) but to different phonemes for speakers of another language. The dictionary must therefore map a word to the possible sequences of phones corresponding to its different pronunciations. In order to reduce complexity, similar phones can be merged and considered as the same phone, however this must done carefully since it reduces the ability to discriminate between similar words. 
An acoustic model is necessary to model the characteristics of a unit of speech, such as phones; in Sphinx, and in most of the current speech recognizers, this is done using Hidden Markov Models. A phone has a typical duration of a few hundreds of milliseconds and is typically divided in three phases: an onset, a middle and an end. Therefore, a simple linear Hidden Markov Model with 3 states is used to model it.

Given the small number of phones present in all of the human languages, it would be very easy to perform speech recognition if they could be used directly. Unfortunately, this is not possible: because of how speech is produced, the same phone in different contexts sounds differently. Therefore, a triphone model is used: combining the models for single phones, we build larger models formed with sequences of three phones. However, this causes an exponential growth of complexity. In order to try to reduce the total number of HMM states needed, a procedure known as state tying is performed: states that are similar according to some statistical measurements are unified into a single state. Finally, HMMs for words are created by assembling those for triphones.

Each state in these HMMs has a mixture of Gaussians output. When using n Gaussians in the mixture, we need to store $n$ values for the means and $n^{2}$ values if a full covariance matrix is used, or $n$ values if only the diagonal is used (i.e. assuming independence). Sphinx defaults to using 8 Gaussians, since experiments performed with a larger number (25) did not yield significant improvements, at the expense of a much larger time needed for computation. 


\subsubsection{Decoder}

The Decoder invokes a SearchManager on the graph provided by the Linguist to score Features from the FrontEnd. The SearchManager can perform different kinds of search, using for example the Viterbi algorithm or other techniques, such as $\mathrm{A}^{*}$. It employs a standard architecture composed of an ActiveList, a Scorer and a Pruner. The ActiveList defines which search states are currently active. The Scorer provides a score for a specific feature vector against a specific HMM state at a given time. The Pruner can remove unpromising paths in the search space through either relative or absolute beam pruning. 


\section{CHAPTER 3}

\section{RELATED WORK}

The only application of RISq (11) so far was in the activity recognition domain $(10 ; 26)$. In this work 8 different activities were used for training and testing reaching an accuracy of $100 \%$ in ideal conditions. In order to improve robustness to occlusion, the human body was decomposed in 5 parts: head and torso, arms and legs. The position and speed of head and torso, upper and lower arms and upper and lower legs were tracked, using a total of 18 dimensions. Votes were collected independently for each body part, so that recognition rates of $70 \%$ to $97.5 \%$ were obtained even with up to 3 out of 5 occluded body parts.

Vector Array Recognition by Indexing and Sequencing (VARIS) is a method that has been recently developed in our lab. It applies the same indexing and sequencing concept of RISq to images to perform Multiple View Face Detection (MVFD) in unconstrained environments (27). VARIS is designed to find the optimal similarity matching between the input image and stored exemplars while allowing wide geometrical variations that are limited only by topological constraints. Aggregated similarity is further enhanced by matching the input images with compound exemplars. The novel compounding procedure also reduces the number of exemplars necessary for each class representation. VARIS with compounding performs efficient parallel classification and has polynomial computational complexity. 


\subsection{HMM-based techniques}

Hidden Markov Models were first proposed in the mid-60s in a series of classical papers from Baum (3) and used for speech recognition in the mid-70s to build the IBM DRAGON system (28). Since then, HMMs became the de facto standard for several pattern recognition tasks, such as speech (4), gestures (29) and human actions (30). However, in order to reach the current level of performance, they underwent an increasing number of modifications and optimizations, which translated into an increasing complexity.

SPHINX (22) is a speech recognition system based on HMMs, developed by Carnegie Mellon University over the last two decades, and is currently considered the state-of-the-art. In tests from Walker et al. SPHINX-4 reaches a $99 \%$ accuracy on a dictionary of up to 100 words, $97 \%$ on a dictionary of up to 1000 words, $93 \%$ on a dictionary of 5000 words and $81 \%$ on a dictionary of 64000 words. However, while the two former results can be achieved in real time, the two latter ones cannot. We have been unable to achieve similar high recognition results with the data that we have been using.

HTK $(31 ; 32)$ is a toolkit to build HMMs for speech recognition, developed at Cambridge University starting in 1989; it is also considered a state-of-the-art system.

Many extensions to HMMs have recently been proposed, the most important being Hierarchical Hidden Markov Models (HHMMs) and Layered Hidden Markov Models (LHMMs). Both extensions focus on the need for less training data with respect to traditional HMMs.

HHMMs (33) propose a recursive model where each state of the HHMM is an HHMM itself. Therefore states emit sequences of symbols instead of a single symbol; in particular, only pro- 
duction states are allowed to emit symbols. HHMMs can be transformed into regular HMMs, however they are in certain cases more efficient than HMMs, at the expense of an increased complexity in the basic algorithms.

LHMMs (34) use $\mathrm{N}$ levels of HMMs where the HMMs on level $\mathrm{N}+1$ corresponds to observation symbols or probability generators at level $N$. Every level $i$ of the LHMM consists of $K_{i}$ HMMs running in parallel. LHMMs can also be transformed into regular HMMs, however they present some advantages with respect to HMMs, such as the need for less training data, due to the fact that each layer can be trained independently of other layers.

HMMs and their extensions just discussed are all generative models, i.e. they use Bayes' rule to compute the posterior probability $\mathrm{P}(M \mid D)$ by estimating the likelihood $\mathrm{P}(\mathrm{D} \mid \mathrm{M})$ and the prior $\mathrm{P}(M)$ from the data. In contrast, discriminative models directly try to estimate $\mathrm{P}(\mathrm{M} \mid \mathrm{D})$ directly from the data. Discriminative models have some advantage on generative model and they have proved to perform better in some domains (35). Support Vector Machines (SVMs) and Neural Networks (NN) are two common examples of discriminative methods. More recently, there has been interest in Maximum Entropy Markov Models (MEMMs) and Conditional Random Fields (CRFs), both of which are also related to HMMs.

MEMMs (36) combine HMMs and the maximum entropy framework (37; 38), allowing observations to be represented as arbitrary overlapping features and defining the conditional probability of a state given the previous state and the current observation, using logistic regression. CRFs (39) are a generalization of Markov Random Fields (MRFs), which in turn are an extension of HMMs for multiple random variables. Differently than all of the previously discussed 
techniques, CRFs are based on an undirected graphical model and were introduced to fix the label bias problem of MEMMs, i.e. the fact that the transitions leaving a given state compete only against each other, rather than against all other transitions in the model. This allows CRFs to make more accurate predictions at the expense of a slower training.

\subsection{Exemplar-based techniques}

There has been increasing interest in recent years in exemplar-based (also called templatebased) techniques for speech recognition.

DeWachter et al. (8) propose a framework that uses many of the typical components of HMM systems, while performing recognition from example feature vectors, instead of building a model for the speech. They substitute the HMM search algorithm with a simpler DTW-based approach, which uses local distance measures and a bottom-up template selection algorithm to keep the search space within reasonable bounds. While their system alone does not outperform HMMs on the chosen task, it leads to improvement on HMM alone when it is combined with a traditional HMM classifier.

Gemmeke et al. (9) focus on how to improve speech recognition over HMM in noisy conditions. They use time-frequency patches as examples, where each patch is the concatenation of several feature vectors. A speech signal is then classified through a sparse representation which is a linear combination of noise and speech patches. Their sparse classification algorithm is based on a typical HMM framework, however it estimates likelihoods of states by weights of activated exemplars rather than with conventional Gaussian Mixture Models (GMM). The system shows promising results, despite not always being better than some baseline recognizers. 


\section{CHAPTER 4}

\section{METHODOLOGY}

In this chapter we will first give a detailed description of how RISq works as a generalpurpose algorithm for temporal pattern recognition, both on isolated sequences and continuous data streams. Then we will describe what modifications are needed to use RISq for speech recognition.

\subsection{Basic algorithm}

RISq is a non-parametric technique, so it does not make any attempt at building a model for each class to be recognized. Therefore, there is no proper training procedure, in the sense that there are no parameters to be learned. Instead, training is performed by simply storing one or more example sequences per class in an underlying data structure. Each sample in a sequence is a vector, whose kind and dimensionality varies with the application. For some applications it might be possible to directly use raw data, whereas with other ones, such as speech, preprocessing is necessary to extract features.

After storing the training data, an unknown input sequence can be classified using a two-step algorithm. The first step is indexing, which consists in identifying the training samples closest to each input sample and assigning them a vote dependent on their distance. The second step is sequencing, which finds a maximum weighted bipartite graph matching between the input sequence and a training sequence, respecting an additional temporal constraint. 
The procedure just described can be applied to isolated sequences, such as single words or a short video containing just one activity. However, additional steps are necessary when an input signal contains several sequences, such as continuous speech or a video containing multiple activities. In the next sections we will first describe in detail the training procedure, then the basic methodology for isolated sequences and the extension to continuous data streams.

\subsection{1 $\quad$ Training}

The training procedure simply consists of storing all the samples from the training sequences in an underlying data structure, along with labeling data, such as its timing, class and sequence identifier. This procedure can be performed with one or more multiple independent sequences per class, an approach that cannot be used with HMMs or DTW.

In the classification stage we retrieve the training samples that are closest to some of the samples from the input sequence, according to some distance function. In order to support this task we need a data structure that allows either efficient k-nearest neighbor retrieval or a range search, i.e. a query of the point inside a certain range.

When operating in more than a few dimensions, the feature space is sparse. In the previous version of RISq, this space was quantized. The sparseness was then exploited by using hash tables to only store the existing data points, instead of explicitly storing a full D-dimensional matrix. This was accomplished with the use of keys formed by concatenating indexes of bins along each dimension. However, this data structure did not allow to efficiently identify neighboring bins, so that the time complexity of the retrieval was $\mathrm{O}(\mathrm{n})$, the same as brute-force linear search. 
In our current approach, we improved on this by using a kd-tree (k-dimensional tree) (40), an extension of a binary tree to multiple dimensions. This is the most efficient known data structure for exact nearest-neighbor retrieval in multiple dimensions. A kd-tree can be built in $\mathrm{O}(n \log (n))$ time and $\mathrm{O}(\mathrm{n})$ space. An example of kd-tree for two dimensions is given in Figure $4^{1}$. At each level of the tree, we split on a different dimension and keep repeating this process until we can organize all of the data points.

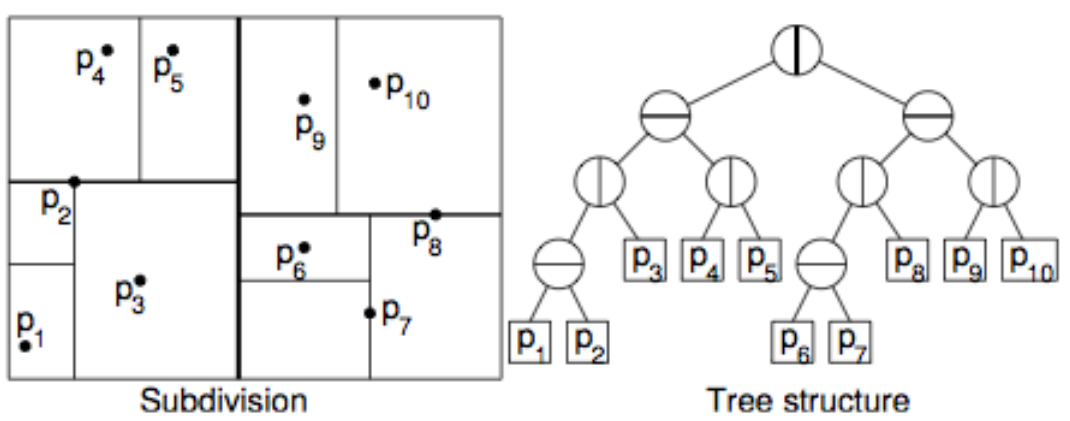

Figure 4. Example of 2D-tree

${ }^{1}$ Copyright, David M. Mount, 2005, Dept. of Computer Science, University of Maryland, College Park, MD, 20742 
If $\mathrm{n}$ points are stored in a balanced kd-tree, the computational complexity of a k-nearestneighbor or a range search is $\mathrm{O}\left(\mathrm{n}^{1-1 / \mathrm{d}}+\mathrm{p}\right)$, where $\mathrm{p}$ is the number of points returned (41). This result holds if $n \gg 2^{\mathrm{D}}$, where $\mathrm{D}$ is the number of dimensions, otherwise most of the tree needs to be searched and the complexity is no better than exhaustive search. However, even the kd-tree cannot escape the so-called "curse of dimensionality". As the number of dimension increases the improvement over a linear search becomes more and more modest.

In order to further improve efficiency, it is necessary to relax the requirement for exact nearestneighbor retrieval. Data structures for approximate nearest-neighbor retrieval are based on multiple randomized kd-trees (42) or hierarchical k-means trees (43). By properly tuning the parameters of either of these algorithms depending on the data set, it is possible to reduce the complexity of the retrieval by one or two orders of magnitude with respect to linear search (44), depending on the desired accuracy. This is not currently part of our implementation.

\subsubsection{Classification of isolated sequences}

The classification procedure comprises two main steps: indexing and sequencing.

In the first step each input sample is indexed in the underlying data structure: the k-nearest neighbors of each input sample according to some distance function are retrieved and assigned a vote depending on their distance from the input sample. An example of how the retrieval process works for a 2-dimensional kd-tree is given in Figure $5^{1}$. Note that it is possible to prune the tree branches which are disjoint from the specified range.

\footnotetext{
${ }^{1}$ Copyright, David M. Mount, 2005, Dept. of Computer Science, University of Maryland, College Park, MD, 20742
} 


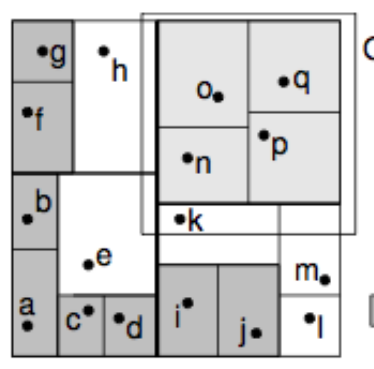

kd-tree subdivision

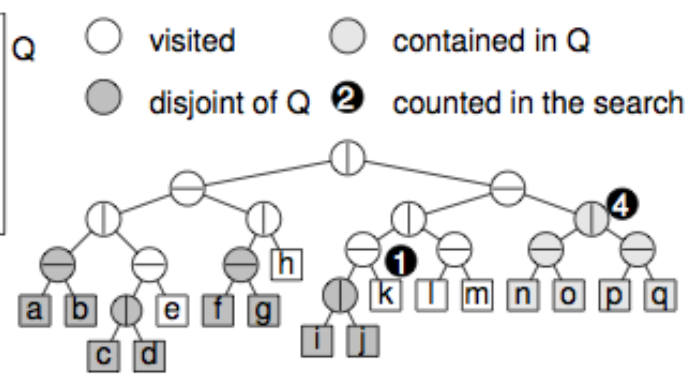

Nodes visited in range search

Figure 5. Example of range search on a 2D-tree

In our experiments, performing a k-nearest neighbor search yielded a better recognition rate than a range search. Moreover, since a k-nearest neighbor search always returns a fixed number of neighbors, it leads to a constant factor in the time complexity of the algorithm, whereas a range search leads to a variable factor. We also experimented with a few distance functions, such as Euclidean and Mahalanobis distance and cosine similarity (not a proper distance function in the mathematical sense). All tests lead to better recognition rates with Euclidean distance. The vote is taken proportionally to a Gaussian function of the distance: vote $=e^{-\mathrm{d}^{2} / 2 \times \sigma^{2}}$ where $\sigma$ is a parameter. This leads to votes between 0 and 1 . Note how the indexing stage is parallel since it considers all of the samples from each example sequence and 
class at the same time, ignoring the class label and timing attached to each sample that are taken into account during the sequencing stage.

The second step in the classification is to find an optimal sequence of votes for each class, that is a maximum weighted bipartite graph matching between the samples of the input sequence and the retrieved samples of each training sequence. This problem is efficiently solvable by the Hungarian method (45). However, we apply an additional temporal sequencing constraint that imposes the same ordering on the matched training sequence as that of the input sequence. Thus, the standard weighted bipartite graph matching no longer applies and we need to develop a novel algorithm to efficiently solve this problem. Let us show an example.

We will first consider the case where training is performed with only one example sequence for each class. Assume that we are computing an optimal sequence for class $i$ and let us define an input sample as $t_{p}$ and a training sample as $n_{i, q}$, where $p$ and $q$ represent timing. Then the constraint is: if $t_{a}$ votes for $n_{i, c}$ and $t_{b}$ votes for $n_{i, d}$ and $a<b$, then $c<d$. An example of this procedure is shown in Figure 6.

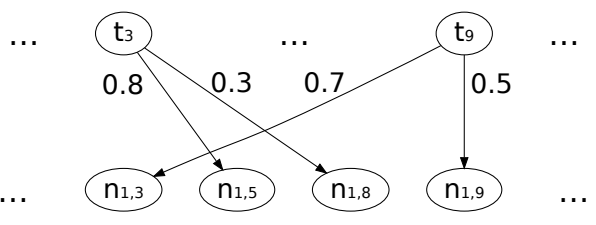

Figure 6. Sequencing example 
Let us compute the matching score between the input sequence and class 1 . At the top of the figure we see two samples from the input sequence, $t_{3}$ and $t_{9}$. At the bottom we see training samples voted by the two input samples from the example sequence for class 1 . The numbers on the edges are votes. The maximum matching is $\left\{t_{3}, n_{1,5}\right\},\left\{t_{9}, n_{1,3}\right\}$, with a score of 1.5. However, this is not allowed because $t_{3}$ precedes $t_{9}$, but $n_{1,5}$ follows $n_{1,3}$. So the optimal matching that respects the temporal constraint is $\left\{t_{3}, n_{1,5}\right\},\left\{t_{9}, n_{1,9}\right\}$, with a score of 1.3 .

When classifying an input sequence, we need to compute an optimal sequence for each trained class, with the following algorithm: at each training sample for that class voted by each input sample, we save the optimal sequence ending at that training sample. In order to efficiently find optimal sequences, we can apply a dynamic programming approach, since this problem exhibits optimal substructure. The optimal sequence ending at some training sample $n_{i, d}$ is formed by the vote towards that training sample and the optimal sequence ending at some previous training sample $n_{i, c}$ that does not violate the temporal constraint (if one exists). Therefore at each training sample, we can save the optimal sequence ending with that sample and look it up later (memoization). This greatly simplifies the process of obtaining optimal sequences. Pseudocode of the classification procedure is in Figure 7.

When training is performed with multiple examples per class, sequencing is performed separately for each example of each class. Subsequently there are two options: either choose the exemplar with the best score or merge the votes from different exemplars. 


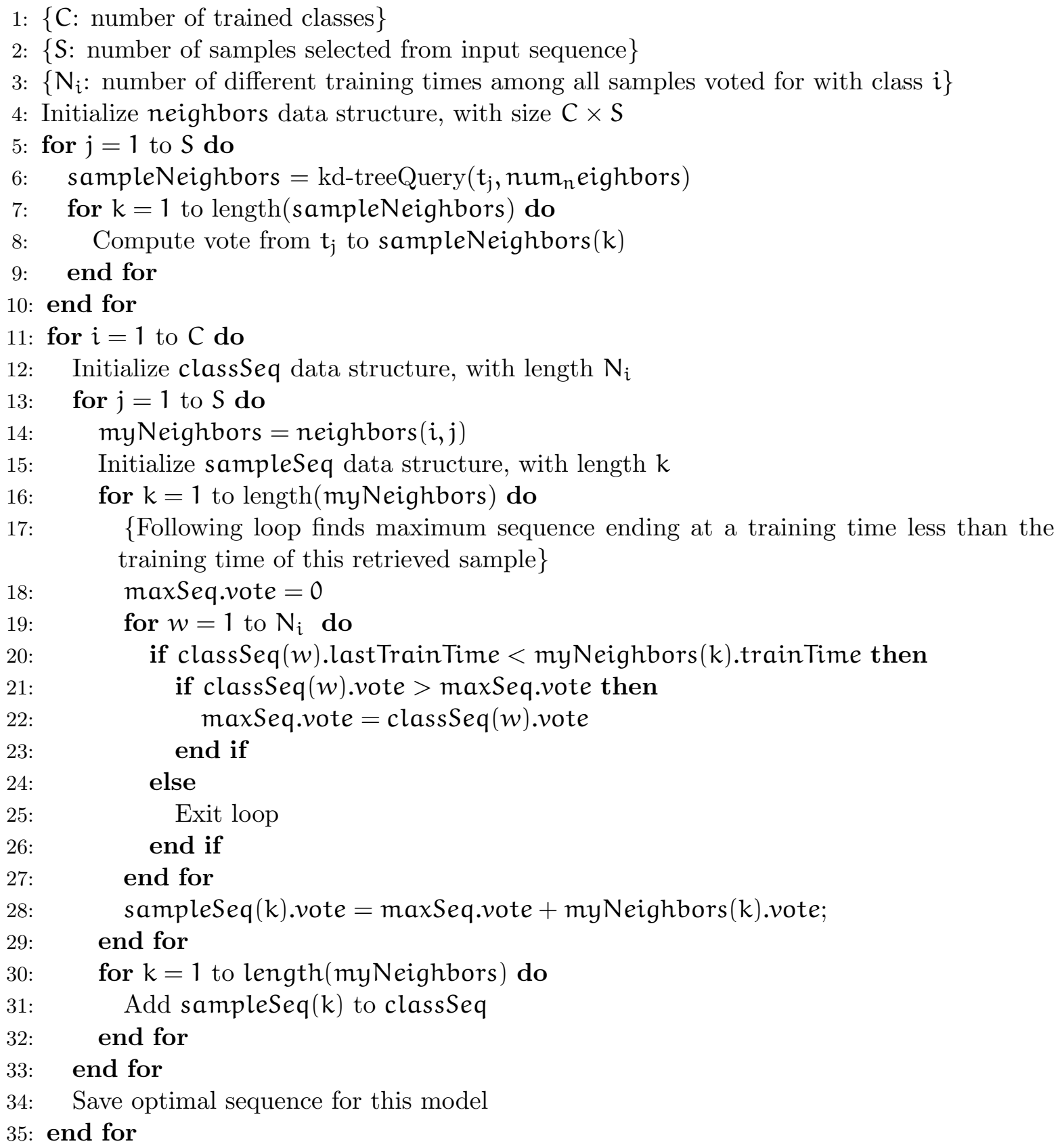

Figure 7. Pseudocode of indexing and sequencing 
Once this process is completed, the final vote for each class is given by the sum of votes in the optimal sequence and the input sequence is classified according to the class with the highest vote.

The analysis of the algorithm in Figure 7 reveals that the most relevant part of the algorithm with respect to time complexity is the sequencing stage. The execution time is directly proportional to the number of trained classes $C$, the number of samples $\mathrm{S}$ selected from the input sequence, the number of retrieved neighbors per sample $\mathrm{T}$ and the number of different training times among all samples voted for with class $i$, which we will call $N_{i}$. Therefore the worst case temporal complexity of the algorithm is $\mathrm{O}\left(\mathrm{C} \times \mathrm{S} \times \mathrm{T} \times \max _{i} \mathrm{~N}_{i}\right)$. This is an improvement over the previous implementation of RISq, which did not use dynamic programming. In that version, the worst case temporal complexity was $\mathrm{O}\left(\mathrm{C} \times \mathrm{S}^{2} \times \mathrm{T}^{2}\right)$.

As can be seen, even with dynamic programming, the temporal complexity still remains linear in the number of classes. Depending on the application, this might not be practical with a large number of classes, especially if there are real-time requirements. In HMM-based systems, domain knowledge is used to prune the search space and limit it to the most likely classes. For example, in speech this is accomplished with a language model. While this approach could be applied to RISq as well, it is also possible to use information derived from the parallel voting stage, such as the number and magnitude of votes collected per class. This is not currently implemented, but is ground for future work. 


\subsubsection{Classification of continuous data streams}

So far we have described the RISq methodology as applied to isolated events, such as single words or gestures. However, it is useful for many domains to be able to apply RISq to a continuous data stream, such as video, audio or some other kind of signal. This is known to be much more complex than classification of isolated sequences because we do not know where an event starts and ends in the data stream.

The main idea to adapt RISq to continuous data streams, such as continuous speech, consists of three major steps:

1. Segment the data stream.

2. Score each segment using the methodology for isolated sequences.

3. Post-process the votes from each segment to decide when to emit class labels.

The first step consists in segmenting the data stream. Ideally, each segment would correspond to a single event and the classification on the data stream would not be more complicated than in the isolated case. However, this does not happen in practice as the segmentation task itself is often extremely difficult. In the case of speech, for example, this is due to the fact that many parts of the speech have very low energy, so that an energy-based approach is not optimal, and also to the fact that words are uttered very close to each other, often with no pause between them. In fact, one task at which humans excel is being able to parse out single words, a significant challenge when learning a new language. 
However, RISq is not particularly susceptible to the segmentation algorithm, because it can easily match parts of an event, without needing the whole event for classification. This allows the use of a simple segmentation algorithm, without a large degradation in performance. We are currently employing a technique where the stream is segmented in fixed-length overlapped segments.

After segmentation, each segment is scored against the training classes using the methodology for isolated sequences as described in the previous section.

Finally, it is necessary to post-process the votes from each segment to decide when to emit class labels. This is necessary because many subsequent segments can be part of the same event, so we cannot just insert in the recognized stream many occurrences of the same event, as this would lead to a so-called insertion error. On the other hand, if two (or more) events are covered by only one segment, we will miss one of them, causing a deletion error. Finally, a substitution error happens when an event is misclassified.

This post-processing phase is currently performed with a peak detection technique. This is motivated by the fact that as a segment "slides" through the event, the overlap between the segment and the event will increase up to a maximum and then decrease. Therefore, if we plot the votes from subsequent segments for the corresponding class, the chart will exhibit a peak. When this peak is reached, we will then emit a corresponding class label. An example of the segmentation and post-processing for speech is given in Section 5.3. 


\subsection{Application to speech recognition}

In order to apply RISq to speech recognition, we first need to extract features from the speech signal. This is done in a way similar to the standard MFCCs and Sphinx, as explained in Section 2.3, but with a few differences. A summary of our procedure is given in Figure 8 .

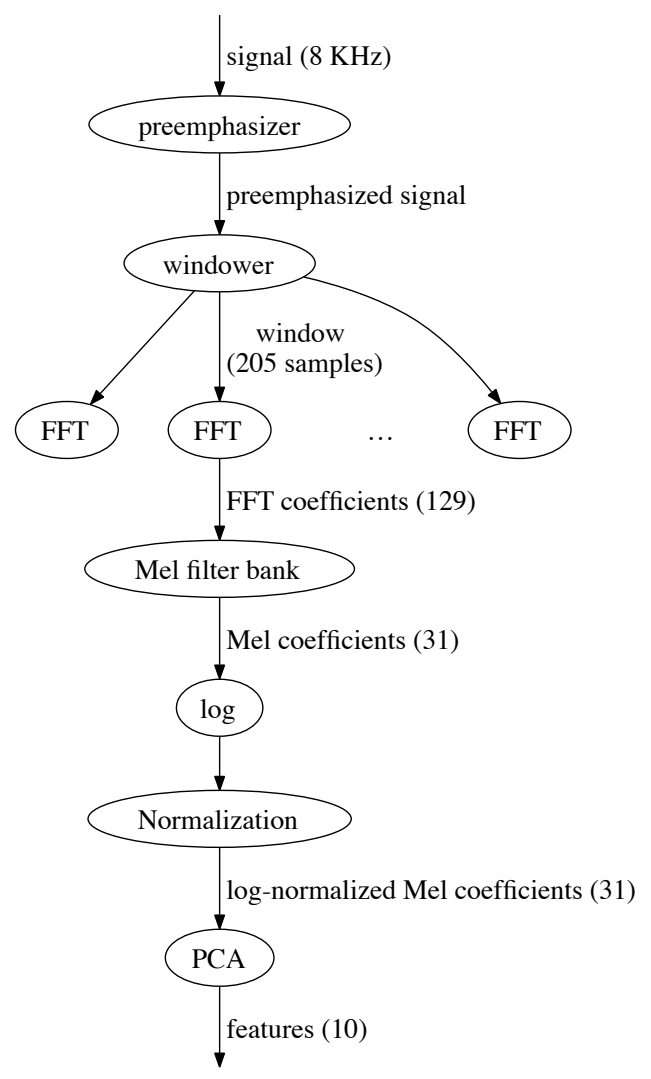

Figure 8. Extraction of features from speech data for RISq 
As can be seen by comparing Figure 8 with Figure 3, the signal processing is the same up to the logarithm of the coefficients outputted by the Mel filter bank. Afterwards, the standard processing proceed to compute a Discrete Cosine Transform (DCT) on these coefficients and retains the first 13 components. This is done to extract the cepstrum, which is basically a smoothed version of the spectrum. After these are computed, cepstral mean normalization is performed. This consists in subtracting the average cepstrum from each resulting vector, which yields cepstrum-normalized MFCC coefficients.

However, with RISq we were not able to achieve sufficient discrimination between words, especially those containing similar sounds, by using standard MFCCs. Using just the coefficients outputted by the Mel filter bank did not yield enough discrimination either. In order to solve this problem, we first normalized each feature vector so that the sum of the squared components is 1 . What this basically does is to disregard the amplitude of the speech signal and conserve only the relative strength at different frequency bands. Subsequently, we applied Principal Component Analysis (PCA) to the training words. PCA is a standard technique used to convert a set of observations of possibly correlated variables into a set of values of linearly uncorrelated variables. The first component is the one which accounts for the highest variability in the data, the second one accounts for the second highest variability and so on. So higher-order components become less and less useful in discriminating between classes. For this reason, we retain only the first 10 components resulting from the PCA. The input data is multiplied by the transformation matrix obtained by the PCA on the training data. 
After the extraction of features, the application of RISq to isolated word recognition follows the methodology for isolated sequences described in the previous section. For continuous speech recognition, we need segmentation and post-processing to decide where to emit words. Moreover, we found that the application of the standard procedure for isolated sequences to the segments could be improved by computing dissimilarities and performing automatic detection of speech parts. In this section we will have a look at the details.

\subsubsection{Segmentation}

While segmentation for speech can be performed by applying a simple fixed-length approach, we found that this does not work well for continuous speech. The main reason is that words can have significantly different lengths. If we take a segment that is too short for a word, it will not give a high enough score. Conversely, if we take a segment that is too long, speech from adjacent words will also likely lower the score.

In order to fix this issue, we process the stream with segments of different lengths. First of all, we determine which segment length to use for each word, depending on the average length of the training exemplars for that word. Then, we run the classification algorithm multiple times, once for each length. While running at a specific length, only the words for which that length was established as optimal are considered for sequencing. Finally, the scores obtained for each length are interpolated in order to run the peak-detection algorithm. While not being perfect, this approach is simple and works better than using a fixed-length approach. Also, as discussed before, there is enough tolerance in the RISq classification to allow for segments that are not exactly the same length as the word to detect, as long as this difference is not too large. 


\subsubsection{Dissimilarities}

When we first applied the procedure for detection of isolated events to the segments, we found more false alarms than initially expected. We eventually traced the problem to the fact that we were only scoring similarities but we did not have any penalization for dissimilarities between the input and the training sequence. This dissimilarity is computed after the sequencing is completed, and can be applied to isolated sequences as well. We are currently using two kinds of dissimilarities. Both are based on the concept that the length of the word segmented from the input stream will roughly match the length of the correct training word.

The first dissimilarity score is proportional to the sum of unmatched samples in the input sequence and in the training sequence.

The second dissimilarity score is based on timing. The training and input sequence are aligned at the midpoint. Afterwards, each vote is re-scored as: $v=v /(1+f * t d)$ where $t d$ is the time difference between the training and input sample that produced this vote and $f$ is a scaling factor. Both input and training sequences have progressive identifiers for the timing, e.g. the

first sample is labeled with 1 , the second with 2 and so on. Therefore the time difference is always an integer number.

While we developed the dissimilarity scores specifically to improve the performance on continuous speech recognition, this approach is completely general and can be used both for the isolated case and for different kind of signals. 


\subsubsection{Automatic detection of parts}

We have developed a novel approach to automatically detect parts in the training words. Once again, while being initially developed for speech, this approach is general and can also be applied to different kind of signals. After the parts are identified, they can be used in different ways. For example, we can equalize the weights on short and long parts or we can set penalties for parts that are not matched. In speech, parts are basically phonemes, i.e. sounds that are the basic components of words. Most phonemes, such as vowels and some consonant classes, are stationary, that is they exhibit the same spectrum as time progresses. This kind of phonemes is easily and mostly correct identified by our approach. However, some classes of consonants are not stationary and our method has more difficulties in identifying them as parts. In particular,

oral stops such as p, b, d, t are not stationary and very short, so they are the hardest to identify. All of the other classes of consonants, affricates (tS, dZ), fricatives (f, v, s, z), nasal stops (m, n) and approximants $(1, r, w, j)$ are easier to identify.

In order to detect parts in the sequences we perform three steps. First we compute autocorrelation on each sequence. Then we detect areas of high autocorrelation at different scales. Finally, we select the best scale that matches each area.

In the first step, we compute autocorrelation of each sequence, that is computing the correlation of each vector with all the other vectors in the sequence. This yields a square matrix whose size is the same as the length of the sequence. An example is given in Figure 9. 


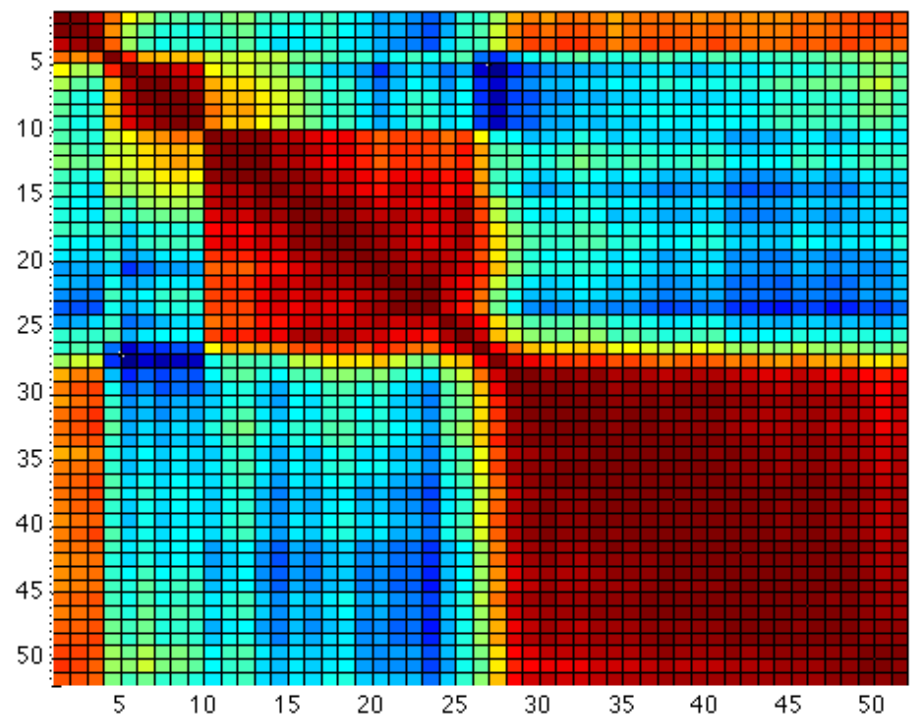

Figure 9. Autocorrelation on the word "glass"

As can be seen, the areas of high correlation are roughly identified by squares along the main diagonal. In order to detect these squares, we convolve the image with square masks of different sizes. If we plot the results of the convolution with masks of different sizes, we can see that peaks are the sharpest as the size of the mask matches the size of the square. An example of this is given in Figure 10. The line at the top corresponds to the smallest mask, size $2 \times 2$. The line at the bottom corresponds to the largest mask, which is $3 / 4$ of the word length. Retaining only the sharpest peak in each region gives the proper scale for each part. 


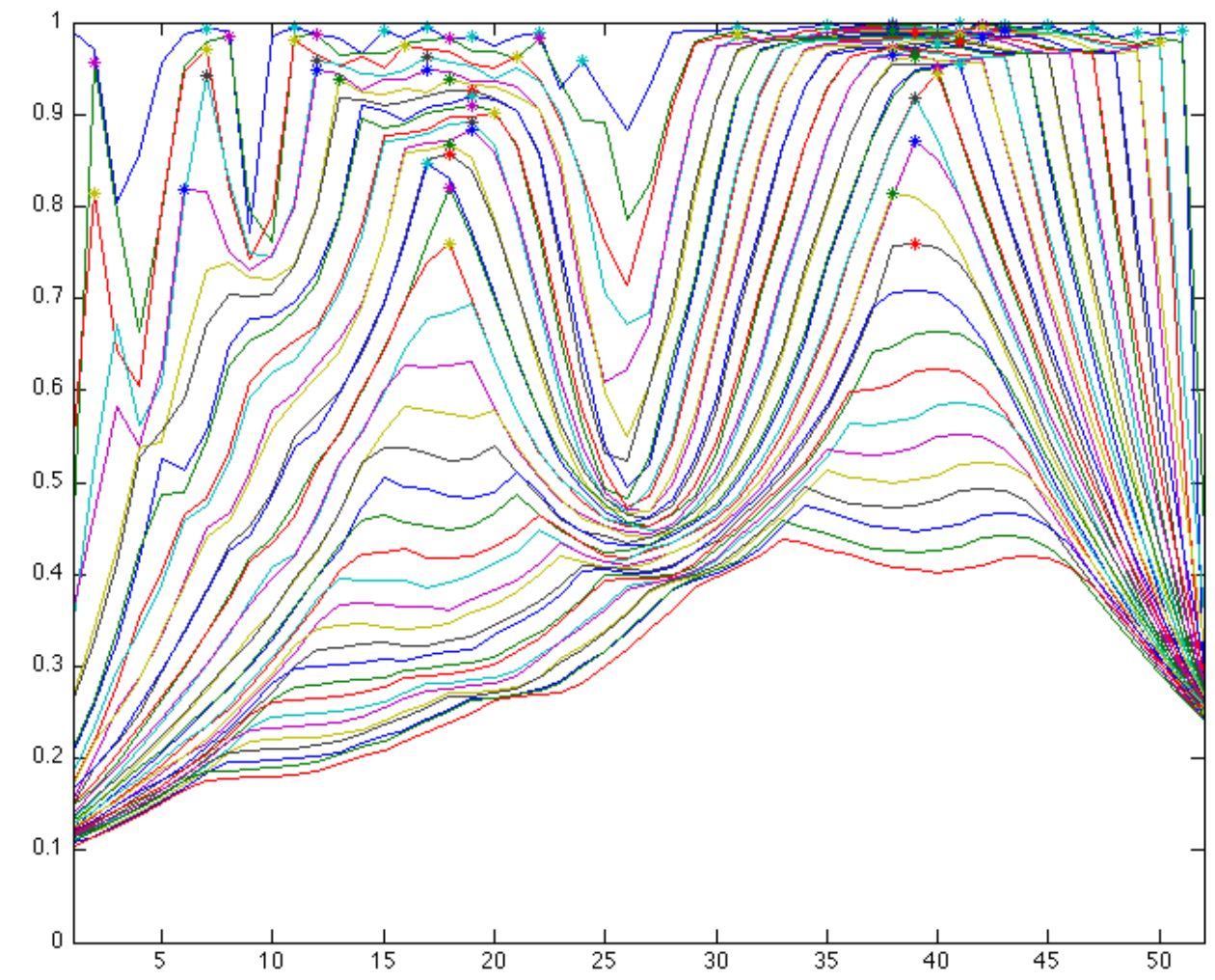

Figure 10. Values of the convolution of the autocorrelation of the word "glass" with masks of different sizes 


\section{CHAPTER 5}

\section{RESULTS}

In this chapter we will provide experimental results of the application of RISq to both isolated and continuous speech recognition on three different corpora, two standard ones and a self-recorded one. In order to validate the performance of RISq, we also compare it to that of Sphinx, a state-of-the-art speech recognizer based on HMMs. Finding an ideal candidate for comparison of RISq is not easy, because of several reasons. First of all, there are very few open source speech recognition systems, of which Sphinx is the most prominent. Also, most of the systems are HMM-based, so they require a very large amount of data for training. Sphinx provides the more feasible alternative of using a few data to adapt existing acoustic models trained on large corpora. Finally, Sphinx is one of the very few complete systems that provide word-level recognition, whereas many of them only provide phoneme-level recognition.

The performance metrics for isolated speech recognition are hit rate and false alarm rate, which can be combined in a Receiver Operating Characteristic (ROC) graph. The metrics for continuous speech recognition are the popular word accuracy and word error rate. A paired sample T-test has been performed to determine whether there is a statistically significant difference on the average recognition rate obtained with RISq and Sphinx.

In the rest of the chapter we will first describe the speech corpora used for evaluation, then present results for isolated and continuous speech recognition. 


\section{1 $\quad$ Speech corpora}

In this section, we describe the speech corpora that have been used for experimental evaluation of the RISq algorithm applied to speech recognition. Similar tests have been conducted with the open source Sphinx software for comparison.

Finding suitable speech corpora to test RISq was also as hard as finding a software to compare with. The ideal corpus would have several speakers repeating the same sentences a few times, since RISq is currently targeting speaker-dependent speech recognition and it is word-based. However, most corpora are geared towards HMM-based systems, which are based on phones and target speaker-independent speech recognition. For this reason, they do not need repetition of the same words from each speaker. As a matter of fact, having the same sentence, or even the same group of words or phonemes repeated many times is not ideal for HMM-based systems. In fact, it is fundamental for these systems to be trained on a corpus that contains phonetically balanced sentences. This means that the sentences need to contain phonetic events according to their frequency of occurrence in natural speech. Corpora to be used with HMM-based systems need to be designed very carefully in order to respect this constraint.

One more issue in finding appropriate corpora to be used with RISq is the dictionary size. When we first started applying RISq to speech recognition we wanted a very small dictionary size, such as ten words. It would have not made sense to move to a larger dictionary size until we could reach reasonably high recognition rates on a smaller dictionary size. Even in the latest stages of development, we are still using a dictionary with only a few dozens words. Finding a corpus that only uses such a small dictionary is very hard. 
Given all the previous reasons, we have performed tests with three different corpora. The Center for Spoken Language Understanding (CSLU) Speaker Recognition corpus $(46 ; 47)$ is the first one that we used for evaluation of isolated-word and continuous speech recognition. A small ten-digit dictionary and several examples of each word from each speaker make this corpus an ideal candidate to be used with RISq and also allow us to investigate the effect of speaker variation on recognition accuracy.

The TIMIT corpus (48) is one of the most popular corpora for evaluation of speech recognition systems. We decided to use this corpus at a later stage to evaluate the performance with a larger dictionary. Labeled time-aligned transcriptions make this corpus very easy to use. However, this database lacks multiple examples of each word from each speaker, thus allowing us to test RISq only in speaker-independent mode.

The Kitchen corpus was inspired by the NSF-funded project "Effective Communication with Robotic Assistants for the Elderly: Integrating Speech, Vision and Haptics" (14). In this project we recorded conversational speech from the interaction of elderly subjects and a helper performing daily activities, such as preparing a meal together. While we initially planned on directly using that data, we realized that there were a few problems. First of all, due to the equipment that we used in the data collection, the final quality of recorded audio proved much lower than expected, leading to poor recognition performance both by RISq and Sphinx. Moreover, the dictionary size is much larger than RISq can currently handle. For these reasons, we decided to record a new corpus with sentences that could be used in the same context as the original corpus but with a smaller dictionary. 


\subsubsection{CSLU Speaker Recognition}

The Center for Spoken Language Understanding (CSLU) Speaker Recognition corpus (46; 47) database includes $8 \mathrm{KHz}$ telephone speech recorded from 91 speakers. Each speaker called an automated system 12 times over 2 years, answering free-speech questions and pronouncing predefined words. From this corpus, we chose 2 predefined 5-digit sequences that each speaker pronounced 4 times during each call: "five three eight two four" and "six one oh nine seven". Thus our dictionary consists of these 10 different digits, the same as in a digit recognition demo provided with the Sphinx system. The reason why we chose these sequences is that our system is word-based, so the training for each speaker needs to be performed using the same words that will be recognized. For training, we manually segmented these sequences in order to isolate the single digits. Note that our procedure does not need perfect segmentation. For example, the inclusion of small silences at the beginning and end of each word does not affect the final result. Given that we used only the data from the first call for each speaker, our dataset consisted of 91 speakers, each pronouncing 10 digits for 4 times, for a total of about 3,500 digits, since a few data are missing from the database.

We used the CSLU corpus for most of the initial development of RISq with isolated word and for part of the development of the continuous speech. Following the initial stage, we wanted to expand the capability of RISq past this very small dictionary and this lead us to consider corpora with a larger dictionary. 


\subsubsection{TIMIT}

The TIMIT corpus (48) was jointly developed by Texas Instruments (TI) and Massachusetts Institute of Technology (MIT) around 1985. It is one of the most popular corpora for evaluation of speech recognition systems. This is because it contains a large amount of data from US speakers from different regions. Moreover, it has time-aligned word and phoneme labeling. The labeling is a very time consuming and error prone process, which is available only for a few databases. The database contains 630 speakers from 8 different dialect US regions. There are 3 different kind of sentences:

- 2 dialect sentences (SA) are pronounced by each speaker.

- 450 compact sentences (SX) are pronounced by 7 speakers each.

- 1890 diverse sentences (SI) are pronounced by only 1 speaker each.

As can be seen, there is no repetition of the same text by the same speaker. While not being a problem for HMM-based systems, this does not allow us to train and test RISq with the same speaker. Therefore, we have used the TIMIT corpus for evaluation of speaker-independent speech recognition, even though RISq is currently not targeted towards this task. In particular, we have used some of the SX sentences. For each sentence, we perform training with 6 speakers and test on the remaining one. We then use n-fold cross-validation. For training, each sentence is automatically segmented at the word level using the time-aligned transcriptions provided with the database. 


\subsubsection{Kitchen}

The Kitchen corpus was inspired by the NSF-funded project "Effective Communication with Robotic Assistants for the Elderly: Integrating Speech, Vision and Haptics" (14). In this project we recorded conversational speech from the interaction of elderly subjects and a helper performing daily activities, such as preparing a meal together. This includes smaller tasks such as retrieving items from the kitchen, collaboratively moving heavy items, cooking and setting the table. The corpus collected in this project is known as Robohelper. Unfortunately, the original data was very noisy due to issues with the equipment that we used for data collection. This heavily affected the performance of both RISq and Sphinx. Therefore we decided to record some new sentences that would be useful for communication in the kitchen, hence the name of the corpus. The idea underlying this decision is to develop a small-dictionary speech recognizer that can be easily trained for each new user. In this context, we anticipate that elderly people will have very significant differences in speech from person to person. Also, it would not be practical to ask them to repeat the same words many times for training.

In order to decide which words to put in our small dictionary, we first ran some statistics on the relative frequency of words in the Find task subset of the original Robohelper corpus. This is a large subset of the corpus that only contains interactions that include a task of finding an object. It contains about 1100 different words and a total of 20700 words. The relative frequency of the words that we selected from the original corpus is given in Table III. As can be seen, the words that we selected cover over $50 \%$ of the total speech. 


\begin{tabular}{|c|c||c|c||c|c||c|c|}
\hline Word & Freq. & Word & Freq. & Word & Freq. & Word & Freq. \\
\hline \hline you & $4.55 \%$ & where & $1.13 \%$ & plate & $0.39 \%$ & silverware & $0.19 \%$ \\
\hline the & $4.43 \%$ & me & $1.03 \%$ & like & $0.38 \%$ & bring & $0.15 \%$ \\
\hline I & $3.66 \%$ & want & $0.90 \%$ & glass & $0.37 \%$ & stove & $0.15 \%$ \\
\hline OK & $3.51 \%$ & put & $0.78 \%$ & soup & $0.33 \%$ & bowl & $0.15 \%$ \\
\hline it & $2.35 \%$ & dont & $0.69 \%$ & table & $0.32 \%$ & dish & $0.08 \%$ \\
\hline to & $2.07 \%$ & on & $0.69 \%$ & bowl & $0.31 \%$ & eat & $0.08 \%$ \\
\hline one & $1.69 \%$ & for & $0.65 \%$ & would & $0.31 \%$ & remember & $0.07 \%$ \\
\hline this & $1.65 \%$ & some & $0.65 \%$ & drawer & $0.31 \%$ & drink & $0.06 \%$ \\
\hline that & $1.64 \%$ & see & $0.62 \%$ & pasta & $0.29 \%$ & very & $0.06 \%$ \\
\hline there & $1.60 \%$ & help & $0.58 \%$ & not & $0.28 \%$ & dinner & $0.04 \%$ \\
\hline can & $1.58 \%$ & over & $0.57 \%$ & salad & $0.27 \%$ & milk & $0.04 \%$ \\
\hline here & $1.52 \%$ & get & $0.56 \%$ & water & $0.26 \%$ & only & $0.04 \%$ \\
\hline we & $1.46 \%$ & have & $0.49 \%$ & could & $0.24 \%$ & clean & $0.02 \%$ \\
\hline in & $1.26 \%$ & need & $0.49 \%$ & spoon & $0.21 \%$ & bread & $0.00 \%$ \\
\hline and & $1.24 \%$ & What & $0.47 \%$ & set & $0.20 \%$ & right & $0.31 \%$ \\
\hline is & $1.21 \%$ & cabinet & $0.42 \%$ & tray & $0.20 \%$ & my & $0.23 \%$ \\
\hline do & $1.19 \%$ & pot & $0.41 \%$ & fridge & $0.19 \%$ & Total & $54.2 \% \%$ \\
\hline
\end{tabular}

TABLE III

\section{WORDS IN THE KITCHEN DICTIONARY AND THEIR RELATIVE FREQUENCIES IN THE ROBOHELPER CORPUS}

This is the list of sentences: "Can you put the glass on the table?", "I would like to eat some pasta", "Bring me the salad and the bread", "I don't drink milk, only water", "Where is the bowl? I don't remember", "It is in the cabinet, not in the fridge", "Could you help me set the table for dinner?", "This dish is not very clean", "Is there soup in that bowl?", "Do we need some silverware?", "OK, I can put it in the drawer", "I have one right here", "What do you want on the tray", "I don't see that pot on the stove", "Can you get that plate over there", "I would like the spoon for my soup".

Each sentence was pronounced by one native and one non-native speaker. 


\subsection{Isolated-word speech recognition results}

Tests for isolated-word speech recognition were performed with the CSLU corpus. In order to assess the performance of RISq and Sphinx on this task, input sequences were classified and results were collected in a multi-class confusion matrix, where rows correspond to actual classes and columns to estimated classes. However, computing statistics from the confusion matrix and performing Receiver Operating Characteristic (ROC) analysis with multiple classes is significantly harder than in the binary case, generally leading to a complexity exponential in the number of classes for an exact solution. Only recently a good approximation has been proposed in the form of a pairwise analysis (49).

Since this kind of analysis is not significant for our application, we follow a more direct approach by computing the number of true positives (TP), true negatives (TN), false positives (FP) and false negatives (FN) on a per-class basis (50). Suppose that we have 3 classes and we want to compute the statistics for class 1 ; we would then regard the values in the confusion matrix as follows:

$$
\left[\begin{array}{lll}
\mathrm{TP} & \mathrm{FP} & \mathrm{FP} \\
\mathrm{FN} & \mathrm{TN} & \mathrm{TN} \\
\mathrm{FN} & \mathrm{TN} & \mathrm{TN}
\end{array}\right]
$$

Similar considerations hold for class 2 and 3. On the basis of these values, we compute four statistics, again on a per-class basis: hit rate $(\mathrm{TP} / \mathrm{TP}+\mathrm{FN})$, false alarm rate $(\mathrm{FP} / \mathrm{FP}+\mathrm{TN})$, positive predictive value $(\mathrm{TP} / \mathrm{TP}+\mathrm{FP})$ and negative predictive value $(\mathrm{TN} / \mathrm{TN}+\mathrm{FN})$. This allows to plot one point in ROC space for each class. In order to compute full ROC curves 
on a per-class basis, we first collect the final votes towards each class for each input sequence and then regard all of the sequences whose real class is the class we are considering as positive examples and all of the sequences whose real class is different as negative examples, as shown above. Full ROC curves for each class are computed using a standard algorithm (51) and averaged to yield one ROC curve for the classifier.

The Real Time Factor (RTF), defined as the ratio of the time needed to process an input sequence to the duration of the sequence, was measured on a MacBook Pro with a $2.4 \mathrm{GHz}$ Intel Core 2 Duo CPU and 4 GB RAM. We used the Sphinx 4 standard Java version and our Matlab implementation for RISq. The kd-tree implementation is in C. We made no major attempt at optimizing the RTF yet.

\subsubsection{Results with RISq}

Features were extracted as described in Section 4.2 and consisted of the 10 components obtained after performing PCA on the logarithm of the output of the Mel filter bank. This processing yields a 10-dimensional vector for each window which constitutes a sample in our sequence. The typical duration of a word in our tests varies from 0.5 seconds to 1 second. This translates to about 50 to 100 samples per word when using the recommended parameter of $25.625 \mathrm{~ms}$ for a window and a gap of $10 \mathrm{~ms}$ between windows.

Training and classification with RISq were performed as explained in Chapter 4: all samples from the training sequences are stored in the kd-tree, whereas only a few samples from the input sequence are used for classification. The main parameters involved in the classification are the re-sampling frequency of the input sequence, the number of nearest neighbors and the $\sigma$ for 
voting. Optimal values of these parameters, determined by statistical analysis, allow to greatly reduce the time needed for classification, while maintaining a high recognition rate.

We setup 3 different experiments. In the first experiment, we trained RISq with some data from one speaker and tested with different data from the same speaker. For the CSLU database, we use 4 repetitions of each digit from each speaker with cross-validation leading to a total of 12 tests per speaker. The average ROC curve obtained from all the speakers is shown in Figure 11. Also, Table IV shows the per-class and average true positive rates when the false positive rate is 0.1 . The RTF was 0.14 .

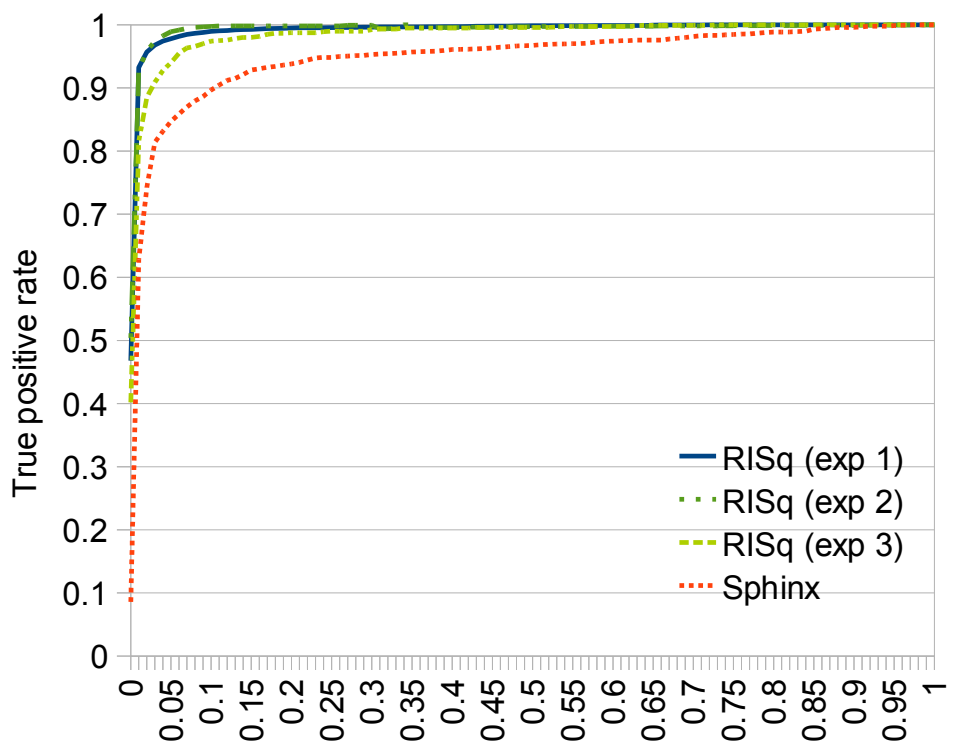

False positive rate

Figure 11. Results on CSLU corpus: average ROCs 


\begin{tabular}{|c|c|c|c|c|}
\hline & RISq1 & RISq2 & RISq3 & Sphinx \\
\hline 0 & .95 & .99 & .99 & .89 \\
\hline 1 & .99 & .99 & .99 & .73 \\
\hline 2 & .99 & .99 & .99 & .98 \\
\hline 3 & .99 & .99 & .99 & .96 \\
\hline 4 & .99 & .99 & .98 & .95 \\
\hline 5 & .99 & .99 & .91 & .87 \\
\hline 6 & .99 & .99 & .90 & .67 \\
\hline 7 & .99 & .98 & .98 & .99 \\
\hline 8 & .99 & .99 & .99 & .93 \\
\hline 9 & .99 & .99 & .99 & .99 \\
\hline \hline avg & .99 & .99 & .97 & .89 \\
\hline
\end{tabular}

TABLE IV

RESULTS ON CSLU CORPUS: PER-CLASS TPR WHEN FPR=0.1

In the second experiment, we trained RISq with multiple examples for each word from multiple speakers and tested with different sequences from the same speakers. For this experiment, we first divided the speakers in groups of 10 and performed cross-validation, again leading to a total of 12 tests per speaker. Results given in Figure 11 and Table IV are extremely similar to those obtained in experiment 1. In particular, the recognition rate for the digit "oh" improved from $95 \%$ to $99 \%$ thanks to the multiple training sequences. The RTF was 0.22 .

In the third experiment, we trained RISq with data from several speakers and tested with data from different speakers. We randomly chose $80 \%$ of the speakers in the dataset for training and the remaining $20 \%$ for testing. The ROC curve in Figure 11 shows that RISq outperforms Sphinx even in this more challenging task. The RTF was 0.30 . 
Note that the flexibility of the training procedure easily allows both speaker-dependent and speaker-independent speech recognition. Experiment 1 is an example of speaker-dependent recognition, which is particularly appropriate when dealing with speech-impaired people or nonnative speakers with heavy accents. Experiments 2 and 3 use speaker-independent recognition and they show excellent results with this small dictionary.

\subsubsection{Results with Sphinx}

The training procedure with Sphinx is more elaborated than it is with RISq and requires a much larger amount of training data. Our dataset was large enough to train and test RISq while collecting statistically significant results, but not for Sphinx. Therefore, we used the standard acoustic models trained with $8 \mathrm{KHz}$ speech from the Wall Street Journal (WSJ) provided with Sphinx. The dictionary consisted of the 10 digits and the language model was a simple grammar with each sentence formed by only 1 of the digits. Even if the WSJ database contains a large number of acoustic models, only those for the 10 words in the dictionary are considered during classification.

The entire CSLU dataset was tested and scored against the WSJ acoustic models. The average ROC curve shown in Figure 11 proves slightly worse than that obtained with the third experiment performed with RISq, where different speakers are used in the training and testing set. Results in Table IV show significant differences in the recognition rate among classes. In particular, recognition of "one" and "six" was significantly worse with Sphinx, possibly due to silence assumptions in the WSJ acoustic models. The RTF was 0.02. 


\subsection{Continuous speech recognition results}

Tests for continuous speech recognition were performed on the CSLU Speaker Recognition, the TIMIT and the Kitchen corpora.

Evaluating the recognition rate of a continuous-word speech recognizer is not straightforward, because the number of words in the utterance and in the recognized string can differ. Therefore it is necessary to first align the reference text and the automatic transcription. In particular, we have used the NIST SClite scoring toolkit, which uses a dynamic programming algorithm performing a global minimization of a Levenshtein distance function which weighs the cost of correct words, insertions, deletions and substitutions as $0,3,3$ and 4 respectively. The computational complexity of this algorithm is $\mathrm{O}(\mathrm{N} \times \mathrm{N})$ where $\mathrm{N}$ is the number of words in the reference text.

After string alignment has been performed, it is possible to compute the Word Error Rate

$W E R=\frac{S+D+I}{N}$ and Word Accuracy WAcc $=\frac{N-(S+D)}{N} . S$ is the number of substitutions, i.e. words which has been misclassified. $\mathrm{D}$ is the number of deletions, i.e. words present in the reference text but not in the automatic transcription. I is the number of insertions, i.e. words present in the automatic transcription but not in the reference text.

The speed of a speech recognizer is usually evaluated with the Real Time Factor (RTF), i.e. the ratio between the time needed to classify an utterance and the duration of the utterance. 


\subsubsection{Results on CSLU corpus}

Tests on the CSLU database for continuous speech were conducted similarly to the ones we did for isolated word. Sample results on a sentence are shown in Figure 12. The top part of the figure shows a sample input sequence "five three eight two four" uttered by speaker 38 .
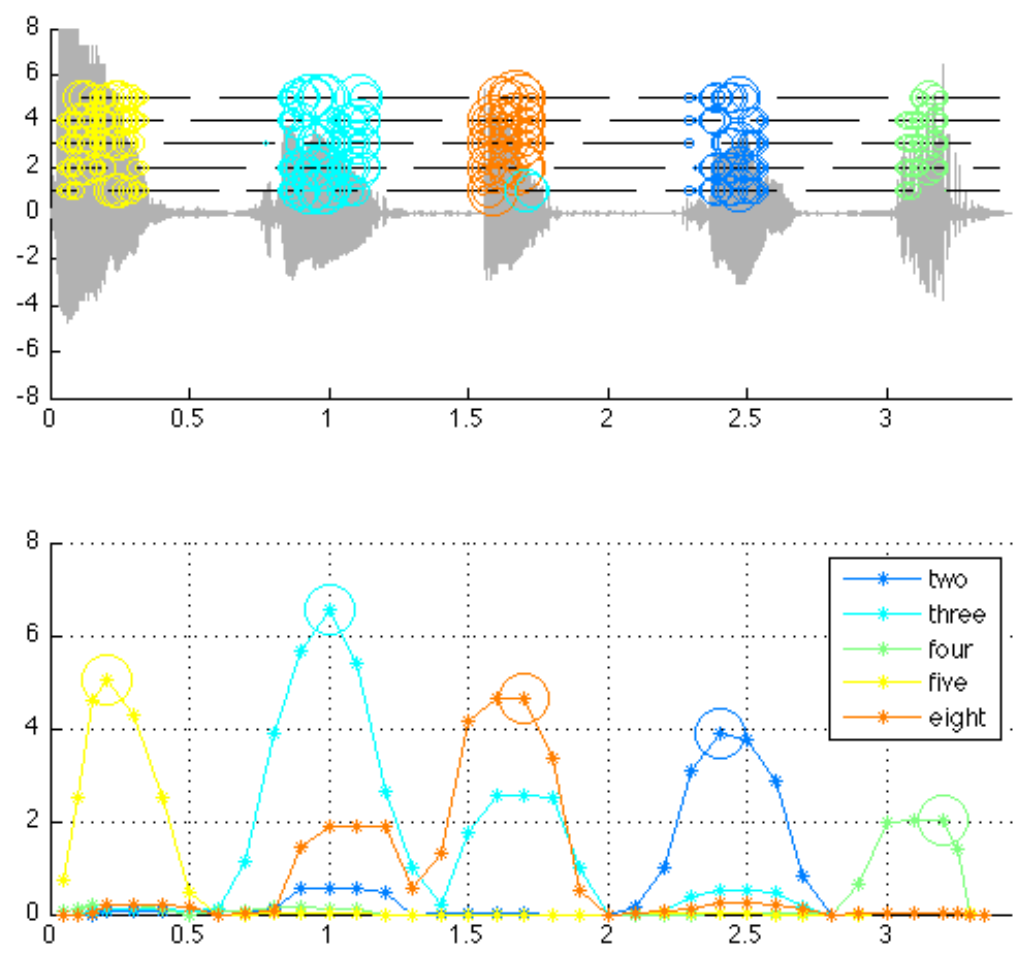

Figure 12. Example of RISq applied to continuous speech 
The training data consisted of a different utterance of the same five words by the same speaker. The figure shows overlapped segments with duration of 0.4 seconds and 0.1 second gap. The circles on each segment represent the testing samples from the input sequence to samples from the training data. Colors identify the winning class for each segment. Therefore we can see that each segment has been correctly classified.

The bottom of Figure 12 shows the final votes for each segment. For easier reference, the votes are plotted aligned with the middle of the segment in the top part of the figure. It is evident the peaking behavior of votes as described in Section 4.1.3. In order to detect peaks we adopt the following algorithm. At each time step, we consider the maximum vote. If the vote is greater than the vote for the same class at the previous and following time steps, then a peak is detected and the corresponding class label is emitted. In this case, the automatic transcription is "five three eight two four" so we do not have any insertion, deletion or substitution error.

We have performed similar tests to those for isolated word from Section 5.2.1. We obtained word accuracy of $96 \%$ for experiment 1 when training with a single speaker and testing on different utterances from the same speaker. Experiment 2 yielded $94 \%$ word accuracy when training with multiple speakers and testing on different utterances from the same speakers. Finally, with experiment 3 we obtained $91 \%$ word accuracy when training with multiple speakers and testing on different speakers. The RTF was 0.5.

We ran tests with Sphinx similarly to what we did for isolated words. We scored the entire CSLU dataset against the WSJ acoustic models and we obtained a word accuracy of $91 \%$. The RTF was 0.2 . 


\subsubsection{Results on TIMIT corpus}

Testing on the TIMIT corpus has been conducted using all the SX sentences from 12 speakers in the TIMIT core test set. Since each speaker pronounces 5 sentences, the total number of test sentences is 60 . For each sentence, training is performed using words from 6 repetitions of the same sentence by different speakers. Also, for each sentence tested, training is performed using only the words that appear in that sentence. Notice that in these tests we ignore the dialect zone of the speakers, so that speakers for each sentence may belong to different dialect areas. The correct recognition rate with RISq was $83.6 \% \pm 7.2 \%$ and the word error rate was $34.3 \%$ $\pm 8.5 \%$. Detailed results for each speaker are in Table V.

\begin{tabular}{|c|c|c|c|c|c|c|}
\hline Sentence & \#Words & Correct\% & Sub\% & Del\% & Ins\% & WErr\% \\
\hline mdab0 & 36 & 72.2 & 2.8 & 25.0 & 22.2 & 50.0 \\
\hline mwbt0 & 37 & 83.8 & 10.8 & 5.4 & 21.6 & 37.8 \\
\hline felc0 & 40 & 87.5 & 5.0 & 7.5 & 30.0 & 42.5 \\
\hline mtas1 & 44 & 81.8 & 6.8 & 11.4 & 18.2 & 36.4 \\
\hline mwew0 & 41 & 90.2 & 0.0 & 9.8 & 14.6 & 24.4 \\
\hline fpas0 & 42 & 69.0 & 9.5 & 21.4 & 9.5 & 40.5 \\
\hline mjmp0 & 36 & 91.7 & 5.6 & 2.8 & 19.4 & 27.8 \\
\hline mlnt0 & 40 & 90.0 & 7.5 & 2.5 & 15.0 & 25.0 \\
\hline fpkt0 & 39 & 87.2 & 7.7 & 5.1 & 20.5 & 33.3 \\
\hline mlll0 & 36 & 77.8 & 2.8 & 19.4 & 19.4 & 41.7 \\
\hline mtls0 & 37 & 86.5 & 10.8 & 2.7 & 13.5 & 27.0 \\
\hline fjlm0 & 36 & 86.1 & 5.6 & 8.3 & 11.1 & 25.0 \\
\hline \hline Mean & 38.7 & 83.7 & 6.2 & 10.1 & 17.9 & 34.3 \\
\hline S.D. & 2.7 & 7.2 & 3.3 & 7.8 & 5.6 & 8.5 \\
\hline
\end{tabular}

TABLE V

DETAILED RESULTS ON TIMIT CORPUS WITH RISQ WITH SENTENCE-SPECIFIC DICTIONARY 
Testing with Sphinx was again done using the WSJ acoustic models. For each test, we created a grammar that only uses the words from the sequence being tested. The correct recognition rate with Sphinx was $95.1 \% \pm 3.0 \%$ and the word error rate was $5.2 \% \pm 2.9 \%$.

\begin{tabular}{|c|c|c|c|c|c|c|}
\hline Sentence & \#Words & Correct\% & Sub\% & Del\% & Ins\% & WErr\% \\
\hline mdab0 & 36 & 88.9 & 2.8 & 8.3 & 0.0 & 11.1 \\
\hline mwbt0 & 37 & 100.0 & 0.0 & 0.0 & 0.0 & 0.0 \\
\hline felc0 & 40 & 95.0 & 0.0 & 5.0 & 0.0 & 5.0 \\
\hline mtas1 & 44 & 95.5 & 0.0 & 4.5 & 0.0 & 4.5 \\
\hline mwew0 & 41 & 97.6 & 2.4 & 0.0 & 0.0 & 2.4 \\
\hline fpas0 & 42 & 90.5 & 0.0 & 9.5 & 0.0 & 9.5 \\
\hline mjmp0 & 36 & 94.4 & 0.0 & 5.6 & 0.0 & 5.6 \\
\hline mlnt0 & 40 & 95.0 & 2.5 & 2.5 & 0.0 & 5.0 \\
\hline fpkt0 & 39 & 94.9 & 0.0 & 5.1 & 0.0 & 5.1 \\
\hline mlll0 & 36 & 97.2 & 2.8 & 0.0 & 0.0 & 2.8 \\
\hline mtls0 & 37 & 97.3 & 2.7 & 0.0 & 2.7 & 5.4 \\
\hline fjlm0 & 36 & 94.4 & 0.0 & 5.6 & 0.0 & 5.6 \\
\hline \hline Mean & 38.7 & 95.1 & 1.1 & 3.8 & 0.2 & 5.2 \\
\hline S.D. & 2.7 & 3.0 & 1.4 & 3.3 & 0.8 & 2.9 \\
\hline
\end{tabular}

TABLE VI

\section{DETAILED RESULTS ON TIMIT CORPUS WITH SPHINX WITH SENTENCE-SPECIFIC DICTIONARY}

A paired sample T-test confirmed that there is a statistically significant difference on the average recognition rates obtained with RISq and Sphinx at a significance level of 0.05. However, keep in mind that Sphinx is trained with a very large amount of data of American English speakers, whereas RISq is trained only with 6 repetitions of each word. Sphinx is expected to perform better than RISq in this speaker-independent scenario. 


\subsubsection{Results on Kitchen corpus}

Testing on the Kitchen corpus was performed with the sentences described in Section 5.1.3. All the 16 sentences are pronounced 2 times by each of the 2 speakers, for a total of 64 sentences. In the first experiment, for each sentence we perform training using only words that appear in the sentence. The training words are extracted from one repetition of the sentence, whereas the other one is used for testing. An example of the recognition is given in Figure 13. We can see that all of the words are identified correctly and that there are no insertions.

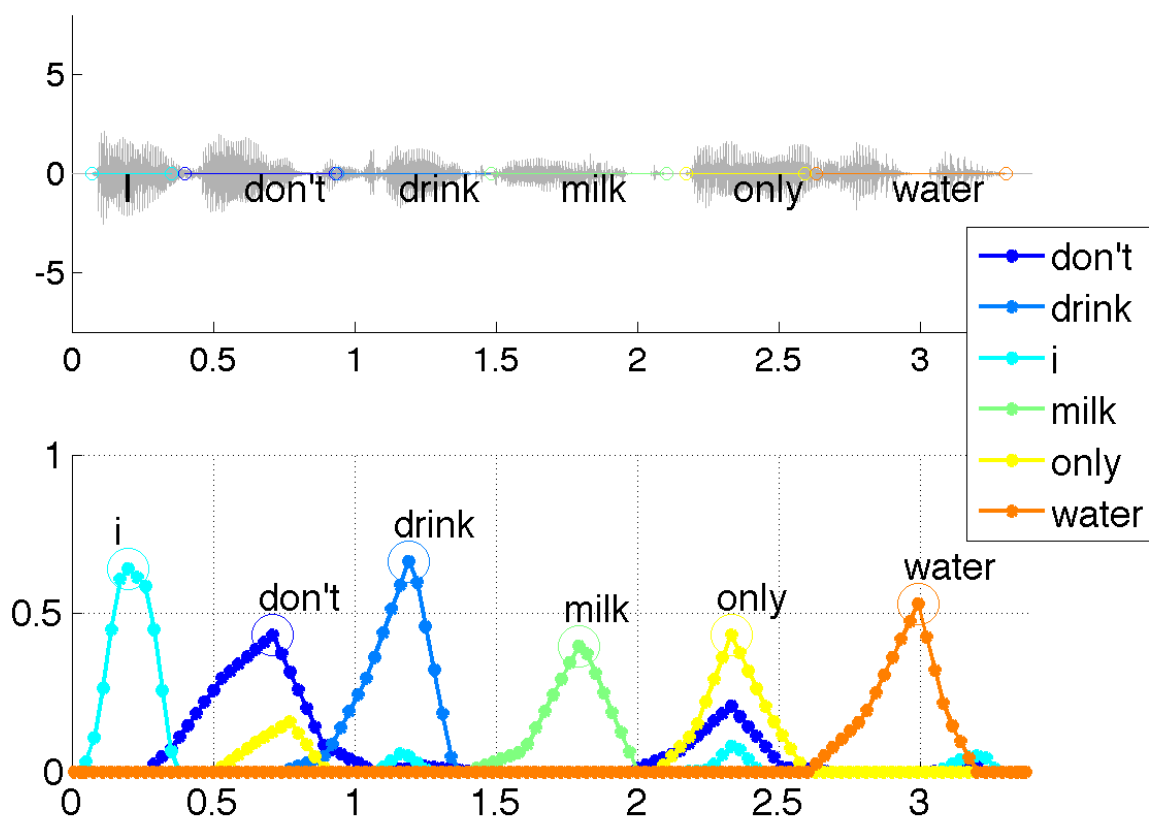

Figure 13. Recognition of a sentence with RISq with sentence-specific dictionary 
Detailed results for each sentence are given in Table VII. Results are given for both the native American English speaker (AE) and the non-native American English speaker (NAE). The correct recognition rate is one minus the sum of substitutions and deletions. The word error rate is the sum of substitutions, deletions and insertions. In the last two rows we also provide mean and standard deviation for each column. The total average correct recognition rate with RISq was $84.1 \% \pm 12.6 \%$ and the word error rate was $21.9 \% \pm 14.8 \%$.

\begin{tabular}{|c|c|c|c|c|c|}
\hline Sentence & \#Words & Corr.\% NAE & Corr.\% AE & WErr\% NAE & WErr\% AE \\
\hline 001 & 8 & 100.0 & 87.5 & 0.0 & 12.5 \\
\hline 002 & 7 & 85.7 & 71.4 & 28.6 & 57.1 \\
\hline 003 & 7 & 85.7 & 71.4 & 28.6 & 28.6 \\
\hline 004 & 6 & 100.0 & 100.0 & 0.0 & 0.0 \\
\hline 005 & 7 & 100.0 & 85.7 & 28.6 & 14.3 \\
\hline 006 & 9 & 88.9 & 66.7 & 22.2 & 33.3 \\
\hline 007 & 9 & 100.0 & 88.9 & 0.0 & 22.2 \\
\hline 008 & 6 & 83.3 & 100.0 & 16.7 & 0.0 \\
\hline 009 & 6 & 100.0 & 66.7 & 16.7 & 33.3 \\
\hline 010 & 5 & 80.0 & 80.0 & 40.0 & 20.0 \\
\hline 011 & 8 & 75.0 & 62.5 & 37.5 & 37.5 \\
\hline 012 & 5 & 100.0 & 100.0 & 0.0 & 0.0 \\
\hline 013 & 7 & 85.7 & 71.4 & 14.3 & 28.6 \\
\hline 014 & 8 & 87.5 & 75.0 & 25.0 & 37.5 \\
\hline 015 & 7 & 71.4 & 71.4 & 28.6 & 28.6 \\
\hline 016 & 8 & 87.5 & 62.5 & 25.0 & 37.5 \\
\hline \hline Mean & 7.1 & 89.4 & 78.8 & 19.5 & 24.4 \\
\hline S.D. & 1.2 & 9.6 & 13.2 & 13.4 & 16.0 \\
\hline
\end{tabular}

TABLE VII

DETAILED RESULTS ON KITCHEN CORPUS WITH RISQ WITH SENTENCE-SPECIFIC DICTIONARY 
Similarly to what we did with the other corpora, we trained Sphinx using the WSJ acoustic models. We also performed adaptation of the acoustic models using one repetition of each sentence and tested on the remaining one. For the first experiment, each sentence was tested with a flat grammar that uses only the words from that sentence. This is a typical setup for such a small dictionary task. Detailed results are given in Table VIII and should be compared with the RISq results in Table VII. The correct recognition rate with Sphinx was $89.2 \% \pm$ $11.3 \%$ and the word error rate was $12.6 \% \pm 13.4 \%$.

\begin{tabular}{|c|c|c|c|c|c|}
\hline Sentence & \#Words & Corr.\% NAE & Corr.\% AE & WErr\% NAE & WErr\% AE \\
\hline 001 & 8 & 87.5 & 87.5 & 25.0 & 12.5 \\
\hline 002 & 7 & 85.7 & 85.7 & 14.3 & 14.3 \\
\hline 003 & 7 & 71.4 & 71.4 & 28.6 & 28.6 \\
\hline 004 & 6 & 83.3 & 100.0 & 16.7 & 0.0 \\
\hline 005 & 7 & 85.7 & 100.0 & 42.9 & 0.0 \\
\hline 006 & 9 & 88.9 & 100.0 & 11.1 & 0.0 \\
\hline 007 & 9 & 55.6 & 88.9 & 44.4 & 11.1 \\
\hline 008 & 6 & 100.0 & 100.0 & 0.0 & 0.0 \\
\hline 009 & 6 & 83.3 & 100.0 & 33.3 & 0.0 \\
\hline 010 & 5 & 100.0 & 100.0 & 0.0 & 0.0 \\
\hline 011 & 8 & 100.0 & 87.5 & 0.0 & 12.5 \\
\hline 012 & 5 & 100.0 & 100.0 & 0.0 & 0.0 \\
\hline 013 & 7 & 100.0 & 85.7 & 0.0 & 14.3 \\
\hline 014 & 8 & 87.5 & 87.5 & 12.5 & 12.5 \\
\hline 015 & 7 & 71.4 & 85.7 & 28.6 & 14.3 \\
\hline 016 & 8 & 75.0 & 100.0 & 25.0 & 0.0 \\
\hline \hline Mean & 7.1 & 86.0 & 92.5 & 17.6 & 7.5 \\
\hline S.D. & 1.2 & 12.9 & 8.6 & 15.5 & 8.6 \\
\hline
\end{tabular}

TABLE VIII

DETAILED RESULTS ON KITCHEN CORPUS WITH SPHINX WITH SENTENCE-SPECIFIC DICTIONARY 
In Table IX we can see a summary of the results for the first experiment. In this experiment, RISq was trained only with the words from each sentence. The dictionary for Sphinx was also limited to only the words appearing in each sentence.

\begin{tabular}{|c|c|c|}
\hline & RISq & Sphinx \\
\hline \hline Corr.\% NAE & $89.4 \pm 9.6$ & $86.0 \pm 12.9$ \\
\hline Corr.\% AE & $78.8 \pm 13.2$ & $92.5 \pm 8.6$ \\
\hline Corr.\% Total & $84.1 \pm 12.6$ & $89.2 \pm 11.3$ \\
\hline \hline WErr.\% NAE & $19.5 \pm 13.4$ & $17.6 \pm 15.5$ \\
\hline WErr.\% AE & $24.4 \pm 16.0$ & $7.5 \pm 8.6$ \\
\hline WErr.\% Total & $21.9 \pm 14.8$ & $12.6 \pm 13.4$ \\
\hline
\end{tabular}

TABLE IX

\section{SUMMARY OF RESULTS WITH SENTENCE-SPECIFIC DICTIONARY}

Informally observing Table IX we can see that Sphinx performed better than RISq on the native American English speaker. In particular, the correct recognition rate was $92.5 \%$ vs. $78.8 \%$ and the word error rate was $7.5 \%$ vs. $24.4 \%$. This was expected, because Sphinx has been trained on a large amount of data of native American English speaker. On the other hand, RISq and Sphinx appear to perform similarly on the non-native American English speaker. Also, RISq performed better on the non-native vs. the native speaker. This is likely due to fact that the native speaker pronounced sentences about $30 \%$ faster than the non-native and had much more audible coarticulation effects. 
In order to evaluate the results in a more formal way, we conducted a one-way withinsentence (repeated measures) Analysis of Variance (ANOVA). In this statistical test, the observed variance within a particular variable is partitioned into components attributable to different sources of variation. This applies to our case, because we are interested in comparing the effects of not only the systems (RISq and Sphinx) but also the speakers (native vs. non-native) on the correct recognition rate and word error rate (the dependent variables). Therefore, performing a simpler T-test would incur increased chance of familywise error (type I error). Each test assumes that the null hypothesis is true, meaning that there are no statistical differences between the means for speaker type by system type. If any one of the four means is statistically different (than what?) we reject the null hypothesis. Statistical significance is achieved when the result of the F-test meets the accepted level of significance (usually 0.05). The F-statistic is the ratio between the explained variance for the variable and unexplained variance. In this context, the explained variance is attributable to the variables under consideration, e.g. the systems or the speakers, whereas the unexplained variance is the total variance of the dataset.

The number of independent variables, as well as the number of samples, influence the shape of the F-distribution which is encompassed in the degrees of freedom. The appropriate degrees of freedom, one for the explained variance and the other for the unexplained variance, are to be used when finding the critical F-value, which is the value that the calculated F-statistic must exceed to meet a specified significance level (in our case, $\mathrm{p}<0.05$ ). 
In our case, we are comparing two levels, or independent variables (speaker by system). The formula for finding explained variance degrees of freedom is the number of levels minus one, so in our case we have 1 degree of freedom in the numerator of the F-statistic. For the unexplained variance, the degrees of freedom are the number of data points in a single group minus one. In our case, we have 16 sentences, leaving us with 15 degrees of freedom in the denominator. The critical value for $\mathrm{F}(1,15)$ in the F-distribution at the level of 0.05 is 4.54 .

When looking at the correct recognition rate, there was not a significant main effect of system $[\mathrm{F}(1,15)=1.14, \mathrm{p}=0.31]$, nor was there a significant main effect of speaker on recognition score $[\mathrm{F}(1,15)=2.87, \mathrm{p}=0.10]$. However, there was a significant interaction of speaker and system on recognition score $[\mathrm{F}(1,15)=12.4, \mathrm{p}=0.01]$. This confirms that one system performs better than the other depending on the speaker, for the reasons that we just explained. In particular, RISq performed better than Sphinx on the non-native speaker and viceversa.

When looking at the word error rate, there was not a significant main effect of the speaker $[\mathrm{F}(1,15)=1.00, \mathrm{p}=0.33]$. There was a significant effect of the system $[\mathrm{F}(1,15)=6.26, \mathrm{p}=0.02]$, as well as a significant interaction of speaker and system $[\mathrm{F}(1,15)=7.23, \mathrm{p}=0.02]$. This result confirms our informal observation that Sphinx does better than RISq in general with respect to word error rate. As we noted earlier, RISq commits quite a few insertion errors (i.e. false alarms), especially due to words which are entirely contained in one another. Also, RISq has some difficulties distinguishing between words that differ only by a consonant, especially if it is a short one like an oral stop (p, t, b, d, k, g). 
In the second experiment, we perform training using all of the words from each sentence. The size of the dictionary obtained in this way is of 67 words, as we explained in Section 5.1.3. An example of recognition on the same sentence as above is given in Figure 14. The words plotted in the figure are only the ones that obtain at least one non-zero vote on one segment. This amounts to only 9 of the total 67 words. As can be seen, with our approach there are a few false detections when a word is entirely contained within another word. In this case we can see detections of the word "on" at the locations of the words "don't" and "only".

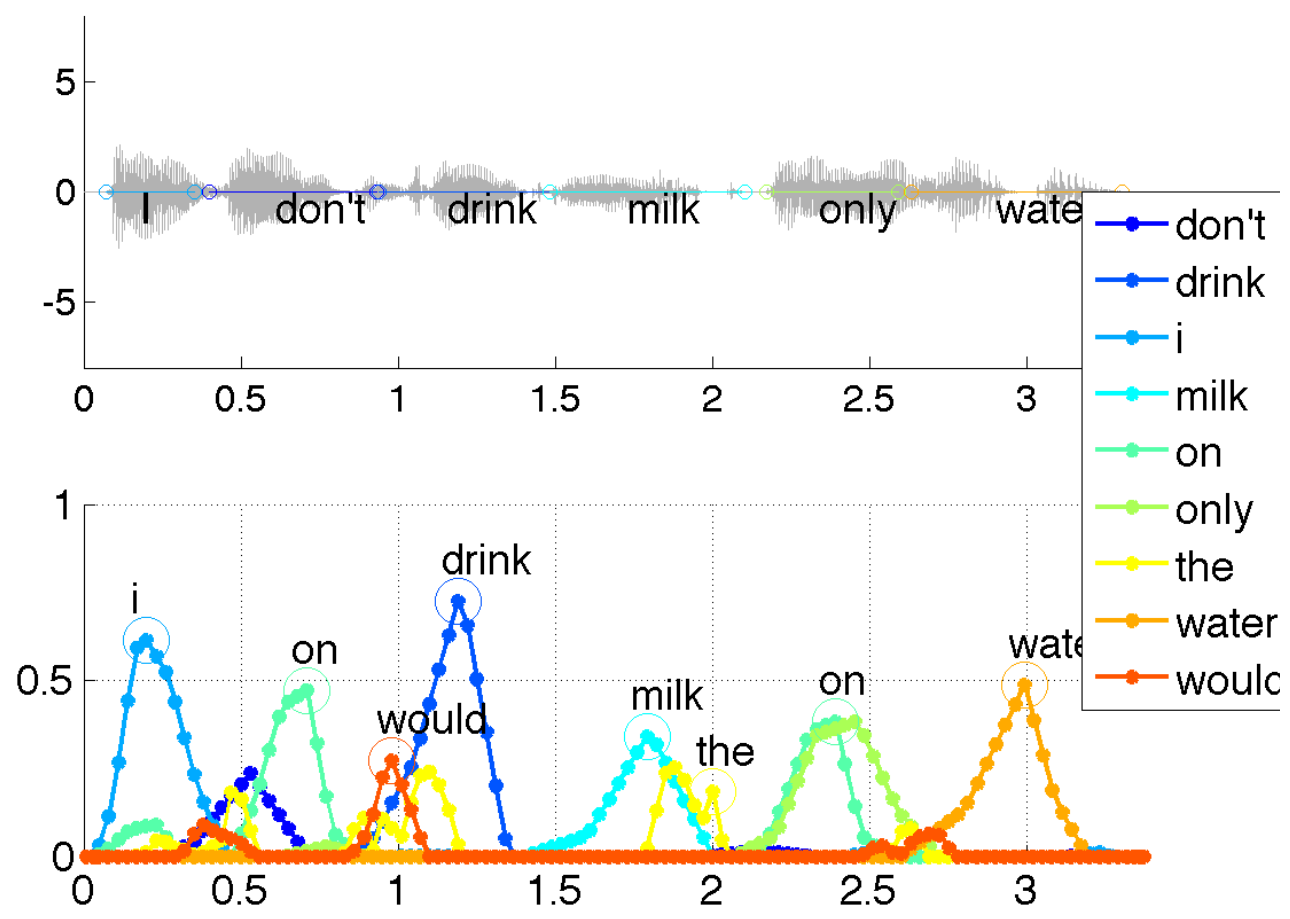

Figure 14. Recognition of a sentence with RISq with full dictionary 
As before, detailed results for this experiment are shown in Table X. The correct recognition rate with RISq was $71.7 \% \pm 17.8 \%$ and the word error rate was $40.0 \% \pm 25.8 \%$. As expected, the correct recognition rate was lower in this experiment with respect to the previous one, because of the expanded dictionary.

\begin{tabular}{|c|c|c|c|c|c|}
\hline Sentence & \#Words & Corr.\% NAE & Corr.\% AE & WErr\% NAE & WErr\% AE \\
\hline 001 & 8 & 100.0 & 87.5 & 0.0 & 12.5 \\
\hline 002 & 7 & 71.4 & 42.9 & 42.9 & 71.4 \\
\hline 003 & 7 & 85.7 & 85.7 & 57.1 & 14.3 \\
\hline 004 & 6 & 66.7 & 100.0 & 100.0 & 0.0 \\
\hline 005 & 7 & 57.1 & 85.7 & 57.1 & 14.3 \\
\hline 006 & 9 & 33.3 & 66.7 & 66.7 & 33.3 \\
\hline 007 & 9 & 88.9 & 88.9 & 11.1 & 22.2 \\
\hline 008 & 6 & 50.0 & 83.3 & 66.7 & 50.0 \\
\hline 009 & 6 & 66.7 & 50.0 & 33.3 & 50.0 \\
\hline 010 & 5 & 80.0 & 80.0 & 80.0 & 20.0 \\
\hline 011 & 8 & 87.5 & 75.0 & 12.5 & 25.0 \\
\hline 012 & 5 & 80.0 & 100.0 & 20.0 & 0.0 \\
\hline 013 & 7 & 71.4 & 57.1 & 57.1 & 57.1 \\
\hline 014 & 8 & 50.0 & 62.5 & 50.0 & 50.0 \\
\hline 015 & 7 & 71.4 & 42.9 & 57.1 & 71.4 \\
\hline 016 & 8 & 75.0 & 50.0 & 25.0 & 50.0 \\
\hline \hline Mean & 7.1 & 70.9 & 72.4 & 46.0 & 33.9 \\
\hline S.D. & 1.2 & 17.0 & 19.5 & 27.3 & 23.6 \\
\hline
\end{tabular}

TABLE X

DETAILED RESULTS ON KITCHEN CORPUS WITH RISQ WITH FULL DICTIONARY 
For the second experiment with Sphinx, we used a uniform grammar that contains all the 67 words in the dictionary. Notice that usually even for such a small dictionary, Sphinx would use a language model to improve recognition. However, since we do not use any language model in RISq, we do not use one with Sphinx either, in order to get a more fair comparison. Detailed results are given in Table XI and should be compared with the RISq results in Table X. The correct recognition rate with Sphinx was $65.2 \% \pm 24.8 \%$ and the word error rate was $47.9 \% \pm$ $41.2 \%$.

\begin{tabular}{|c|c|c|c|c|c|}
\hline Sentence & \#Words & Corr.\% NAE & Corr.\% AE & WErr\% NAE & WErr\% AE \\
\hline 001 & 8 & 37.5 & 87.5 & 87.5 & 12.5 \\
\hline 002 & 7 & 71.4 & 85.7 & 42.9 & 14.3 \\
\hline 003 & 7 & 28.6 & 42.9 & 114.3 & 57.1 \\
\hline 004 & 6 & 16.7 & 100.0 & 183.3 & 0.0 \\
\hline 005 & 7 & 71.4 & 85.7 & 42.9 & 28.6 \\
\hline 006 & 9 & 66.7 & 100.0 & 33.3 & 0.0 \\
\hline 007 & 9 & 33.3 & 77.8 & 100.0 & 33.3 \\
\hline 008 & 6 & 66.7 & 100.0 & 50.0 & 0.0 \\
\hline 009 & 6 & 83.3 & 50.0 & 50.0 & 50.0 \\
\hline 010 & 5 & 40.0 & 80.0 & 120.0 & 20.0 \\
\hline 011 & 8 & 50.0 & 75.0 & 62.5 & 25.0 \\
\hline 012 & 5 & 100.0 & 100.0 & 0.0 & 0.0 \\
\hline 013 & 7 & 57.1 & 71.4 & 42.9 & 28.6 \\
\hline 014 & 8 & 25.0 & 75.0 & 75.0 & 25.0 \\
\hline 015 & 7 & 42.9 & 28.6 & 71.4 & 85.7 \\
\hline 016 & 8 & 62.5 & 75.0 & 50.0 & 25.0 \\
\hline \hline Mean & 7.1 & 53.3 & 77.2 & 70.4 & 25.3 \\
\hline S.D. & 1.2 & 22.9 & 21.1 & 43.4 & 23.4 \\
\hline
\end{tabular}

TABLE XI

\section{DETAILED RESULTS ON KITCHEN CORPUS WITH SPHINX WITH FULL} DICTIONARY 
In Table XII we can see a summary of the results for the second experiment. In this experiment, RISq was trained with the words from all the sentences. The dictionary for Sphinx also contained all the words from each sentence.

\begin{tabular}{|c|c|c|}
\hline & RISq & Sphinx \\
\hline \hline Corr.\% NAE & $70.9 \pm 17.0$ & $53.3 \pm 22.9$ \\
\hline Corr.\% AE & $72.4 \pm 19.5$ & $77.2 \pm 21.1$ \\
\hline Corr.\% Total & $71.7 \pm 17.8$ & $65.2 \pm 24.8$ \\
\hline \hline WErr.\% NAE & $46.0 \pm 27.3$ & $70.4 \pm 43.4$ \\
\hline WErr.\% AE & $33.9 \pm 23.6$ & $25.3 \pm 23.4$ \\
\hline WErr.\% Total & $40.0 \pm 25.8$ & $47.9 \pm 41.2$ \\
\hline
\end{tabular}

TABLE XII

SUMMARY OF RESULTS WITH FULL DICTIONARY

Again, testing with ANOVA on the correct recognition rate found no significant effect of the system $[\mathrm{F}(1,15)=1.84, \mathrm{p}=0.20]$. The effect of the speaker was just slightly not significant $[\mathrm{F}(1,15)=4.30, \mathrm{p}=0.06]$. However, there was a significant interaction of speaker and system $[\mathrm{F}(1,15)=12.53, \mathrm{p}=0.01]$. As before, RISq performed better than Sphinx on the non-native speaker and viceversa, even though the difference is now more evident.

When looking at the word error rate, there was no significant effect of the system $[\mathrm{F}(1,15)=$ 1.19, $\mathrm{p}=0.29]$. There was a significant effect of the speaker $[\mathrm{F}(1,15)=9.00, \mathrm{p}=0.01]$, as well as a significant interaction of speaker and system $[\mathrm{F}(1,15)=13.83, \mathrm{p}=0.01]$. 
Lastly, we conducted an informal comparison with the Google speech recognition system. Because this is not an open system, we cannot control any of its parameters. In particular, there is no way of controlling the dictionary. For this reason, a rigorous comparison with RISq or Sphinx is not easy. Detailed results are given in Table XIII and can be informally compared to the results in Table $\mathrm{X}$ and Table XI. The average correct recognition rate with the Google recognizer was $79.2 \% \pm 26.3 \%$ and the average word error rate was $22.6 \% \pm 29.2 \%$. However, it can be seen how the recognition rate for the non-native speaker was only $69.4 \%$, whereas it was $89.1 \%$ for the native speaker.

\begin{tabular}{|c|c|c|c|c|c|}
\hline Sentence & \#Words & Corr.\% NAE & Corr.\% AE & WErr\% NAE & WErr\% AE \\
\hline 001 & 8 & 100.0 & 100.0 & 0.0 & 0.0 \\
\hline 002 & 7 & 100.0 & 100.0 & 0.0 & 0.0 \\
\hline 003 & 7 & 14.3 & 85.7 & 85.7 & 14.3 \\
\hline 004 & 6 & 33.3 & 100.0 & 100.0 & 0.0 \\
\hline 005 & 7 & 85.7 & 85.7 & 14.3 & 14.3 \\
\hline 006 & 9 & 33.3 & 100.0 & 66.7 & 0.0 \\
\hline 007 & 9 & 77.8 & 100.0 & 33.3 & 0.0 \\
\hline 008 & 6 & 66.7 & 100.0 & 33.3 & 0.0 \\
\hline 009 & 6 & 16.7 & 33.3 & 83.3 & 66.7 \\
\hline 010 & 5 & 100.0 & 100.0 & 0.0 & 0.0 \\
\hline 011 & 8 & 87.5 & 87.5 & 12.5 & 12.5 \\
\hline 012 & 5 & 100.0 & 100.0 & 0.0 & 0.0 \\
\hline 013 & 7 & 100.0 & 85.7 & 0.0 & 14.3 \\
\hline 014 & 8 & 75.0 & 75.0 & 25.0 & 25.0 \\
\hline 015 & 7 & 57.1 & 85.7 & 57.1 & 14.3 \\
\hline 016 & 8 & 62.5 & 87.5 & 37.5 & 12.5 \\
\hline \hline Mean & 7.1 & 69.4 & 89.1 & 34.3 & 10.9 \\
\hline S.D. & 1.2 & 30.6 & 17.0 & 34.4 & 17.0 \\
\hline
\end{tabular}

TABLE XIII

DETAILED RESULTS ON KITCHEN CORPUS WITH GOOGLE SPEECH RECOGNIZER 


\section{CHAPTER 6}

\section{CONCLUSIONS}

Despite decades of research in the area, speech recognition still remains an open problem, mainly because of large intra-speaker and inter-speaker variation. Early research in the area focused on comparing the similarity of training speech signals with an input signal, such as in Dynamic Time Warping (DTW). This technique was later superseded by Hidden Markov Models (HMMs), a more complex parametric technique that dominated the field for the past few decades. However, despite many refinements, HMM-based algorithms did not yield exceptionally high performance. In recent years, there has been increasing interest in non-parametric exemplar-based techniques. Despite showing some interesting results, most of these techniques are still based on a typical HMM-like framework.

The research presented here is based on Recognition by Indexing and Sequencing (RISq), an algorithm initially developed for human activity recognition. As it turns out, RISq takes a very flexible approach that can be applied to any problem involving temporal pattern recognition. Like DTW, RISq computes a similarity score by comparing the input sequence to one or a few exemplars of each class. However, RISq has several advantages with respect to both DTW and HMMs. RISq can achieve robust recognition even when training is performed with only a few exemplar from each class. Additionally, RISq is more robust than HMMs in conditions of noise and missing information and recognition is partially parallel whereas with HMMs or DTW it is completely serial. 
In RISq, training simply consists of storing a few exemplars for each class to be recognized in a data structure for efficient retrieval. Classification is performed in two steps: voting and sequencing. In the voting stage, the $k$ nearest neighbors of each input sample are retrieved. In the sequencing stage, an optimal sequence is built for each class to be recognized through a dynamic programming algorithm. The application of RISq to continuous data streams, such as speech, uses a segmentation approach and a post-processing peak detection algorithm to identify events in the stream.

In this work we presented an improved methodology for RISq and the application of RISq to both isolated-word and continuous speech recognition. While most of the improvements presented here have been developed to cope with the unique challenges of speech recognition, most of them are completely general and can be applied to many other kinds of signals.

When applied to speech recognition, RISq shows some advantages with respect to state-ofthe-art systems. Most speech recognizers are trained on a specific corpus that usually includes only native speakers of a language. Therefore, speakers with heavy accents often experience disappointing performance from such systems, despite the advertised recognition rates. Even if adaptation to a speaker's voice is possible, it is often not enough to let a user naturally interact with it. Due to its simple training procedure, RISq can be quickly adapted to a new speaker, thus effectively enabling speaker-dependent speech recognition. Moreover, being purely based on a pattern recognition approach, RISq can be adapted to any language without any additional work. In traditional speech recognizers, it is necessary to provide a language model, that needs to be computed from a very large amount of data. 
Our results with both the open-source Sphinx system and the commercial Google speech recognizer show how a non-native American English speaker will usually obtain much lower recognition rates than advertised. This is because these systems are trained on very large corpora which are based on native speakers. We showed how with RISq we can improve on these results even by using only one repetition of each word from that same speaker. This approach is not possible with HMMs, as it would require too many data and careful tuning of many parameters by the user. RISq achieves a satisfying performance level without using any domain knowledge. Therefore, it disregards the accent and even the language of the speaker.

There are several areas for improving RISq as applied to speech recognition. With RISq we have moved away from classical Mel-Frequency Cepstral Coefficients (MFCCs) features, in favor of Mel-Frequency Filter Bank (MFFB) coefficients, normalized and passed through Principal Component Analysis (PCA). However, we feel like there is still room for improvement on the kind of features used for speech recognition. For example, Poggio has showed that localized spectro-temporal features (52) have some advantages over MFCCs. Since this kind of features is basically an image, this could be combined with the recently developed Vector Array Recognition by Indexing and Sequencing (VARIS) (27).

Another area for improvement is in the post-processing of votes. The current algorithm for the final emission of words uses a simple peak detection that still yields too many false alarms, especially with words that are entirely contained in other words. This algorithm could be changed with a smarter one. For example, it could be based on some optimization technique to consider the area under each peak and evaluate alternative words. 
The use of a language model would most likely increase recognition performance. It would allow to evaluate different sequences of words and take into account what is more likely to be pronounced. However, this would come at the expense of not relying on pure pattern recognition. It would also require to obtain a language model when adapting RISq to a new language.

Another more radical approach would be to consider phonemes as the basic unit for training, instead of words. This is technically possible without any modification of the basic algorithm. However, similarly to HMM-based systems, it would require a dictionary to map pronunciation of words to phonemes. Moreover, it would require an additional layer to combine phonemes into words.

The application of RISq to speech recognition constitutes a very novel approach to this problem. In this work, we showed how RISq presents both theoretical and practical advantages with respect to now prevalent HMM-based systems, such as Sphinx. In particular we showed how RISq constitutes a competitive approach to a personal speech recognition system. 


\section{CITED LITERATURE}

1. NIST: The History of Automatic Speech Recognition Evaluations at NIST. http://itl.nist.gov/iad/mig/publications/ASRhistory/index.html.

2. Sakoe, H. and Chiba, S.: Dynamic Programming Algorithm Optimization for Spoken Word Recognition. IEEE Transactions on Acoustics, Speech, and Signal Processing, 26:43-49, 1978.

3. Baum, L. E. and Petrie, T.: Statistical Inference for Probabilistic Functions of Finite State Markov Chains. The Annals of Mathematical Statistics, 37:1554-1563, 1966.

4. Rabiner, L. R.: A Tutorial on Hidden Markov Models and Selected Applications in Speech Recognition. Proceedings of the IEEE, 77(2):257-286, 1989.

5. Dempster, A. P., Laird, N. M., and Rubin, D. B.: Maximum Likelihood from Incomplete Data via the EM Algorithm. Journal of the Royal Statistical Society, 39:1-38, 1977.

6. Baker, J., Deng, L., Glass, J., Khudanpur, S., Lee, C., Morgan, N., and OShaughnessy, D.: Research Developments and Directions in Speech Recognition and Understanding, Part 1. IEEE Signal Processing Magazine, 26:75-80, May 2009.

7. Baker, J., Deng, L., Khudanpur, S., Lee, C., Glass, J., Morgan, N., and OShaughnessy, D.: Updated MINDS Report on Speech Recognition and Understanding, Part 2. IEEE Signal Processing Magazine, 26:78-85, July 2009.

8. De Wachter, M., Matton, M., Demuynck, K., Wambacq, P., Cools, R., and Van Compernolle, D.: Template-Based Continuous Speech Recognition. IEEE Transactions on Audio, Speech and Language Processing, 15:1377-1390, May 2007.

9. Gemmeke, J., Virtanen, T., and Hurmalainen, A.: Exemplar-Based Sparse Representations for Noise Robust Automatic Speech Recognition. IEEE Transactions on Audio,

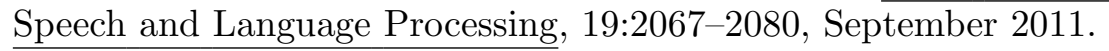

10. Ben-Arie, J., Wang, Z., Pandit, P., and Rajaram, S.: Human Activity Recognition Using Multidimensional Indexing. IEEE Transactions on Pattern Analysis and Machine Intelligence, 24(8):1091-1104, 2002. 
11. Ben-Arie, J.: Method of Recognition of Human Motion, Vector Sequences and Speech. US Patent 7,366,645, April 2008.

12. Franzini, S. and Ben-Arie, J.: Speech Recognition by Indexing and Sequencing. In Proceedings of the International Conference of Soft Computing and Pattern Recognition, pages 93-98, 2010.

13. Franzini, S. and Ben-Arie, J.: Speech Recognition by Indexing and Sequencing. International Journal of Computer Information Systems and Industrial Management Applications, 4:358-365, 2012.

14. Zefran, M., Ben-Arie, J., Di Eugenio, B., and Foreman, M. D.: Effective Communication with Robotic Assistants for the Elderly: Integrating Speech, Vision and Haptics. NSF Grant \#0905593, 2009.

15. Vipperla, R., Wolters, M., Georgila, K., and Renals, S.: Speech Input from Older Users in Smart Environments: Challenges and Perspectives. In Proceedings of the International Conference on Universal Access in Human-Computer Interaction, pages 117-126, 2009.

16. ten Holt, G. A., Reinders, M. J. T., and Hendriks, E. A.: Multi-Dimensional Dynamic Time Warping for Gesture Recognition. In Annual Conference of the Advanced School for Computing and Imaging, 2007.

17. Nair, N. U. and Sreenivas, T. V.: Multi Pattern Dynamic Time Warping for Automatic Speech Recognition. In Tencon, 2008.

18. Baum, L. E., Petrie, T., Soules, G., and Weiss, N.: A Maximization Technique Occurring in the Statistical Analysis of Probabilistic Functions of Markov Chains. The Annals of Mathematical Statistics, 41:164-171, 1970.

19. Velichko, V. M. and Zagoruyko, N. G.: Automatic recognition of 200 words. International Journal of Man-Machine Studies, 2:223-234, 1970.

20. Myers, C., Rabiner, L. R., and Rosenberg, A. E.: Performance Tradeoffs in Dynamic Time Warping Algorithms for Isolated Word Recognition. IEEE Transactions on Acoustics, Speech, and Signal Processing, 28:623-635, 1980.

21. Jelinek, F.: Statistical Methods for Speech Recognition. The MIT Press, 1998. 
22. Walker, W., Lamere, P., Kwok, P., Raj, B., Singh, R., Gouvea, E., Wolf, P., and Woelfel, J.: Sphinx-4: A Flexible Open Source Framework for Speech Recognition. Technical report TR-2004-139, Sun Microsystems, 2004.

23. group, R.: Open Source Tutorial: Learning to Use the CMU SPHINX Automatic Speech Recognition System.

http://www.speech.cs.cmu.edu/sphinx/tutorial.html.

24. Davis, S. B. and Mermelstein, P.: Comparison of Parametric Representations for Monosyllabic Word Recognition in Continuously Spoken Sentences. IEEE Transactions on Acoustics, Speech, and Signal Processing, 28:357-366, 1980.

25. Vertanen, K.: Baseline WSJ Acoustic Models for HTK and Sphinx: Training Recipes and Recognition Experiments. Technical report, Cavendish Laboratory, University of Cambridge, 2006.

26. Sivalingam, D. M.: Analysis of Human Motion: Labeling, Activity and Multi-class Recognition. Master's thesis, University of Illinois at Chicago, Department of Electrical and Computer Engineering, 2003.

27. Ma, K. and Ben-Arie, J.: Vector Array Based Multi-View Face Detection with Compound Exemplars. In Proceedings of the IEEE Conference on Computer Vision and Pattern Recognition, pages 3186-3193, 2012.

28. Baker, J. K.: The DRAGON System - An Overview. IEEE Transactions on Acoustics, Speech and Signal Processing, 23(1):24-29, February 1975.

29. Yang, J. and Xu, Y.: Hidden Markov Model for Gesture Recognition. Technical report CMU-RI-TR-94-10, Carnegie Mellon University, Robotics Institute, 1994.

30. Yamato, J., Ohya, J., and Ishii, K.: Recognizing Human Action in Time-Sequential Images Using Hidden Markov Model. In Computer Vision and Pattern Recognition, pages 379-385, 1992.

31. Woodland, P. C., Odell, J. J., Valtchev, V., and Young, S. J.: Large Vocabulary Continuous Speech Recognition Using HTK. In Proceedings of the International Conference on Acoustics, Speech and Signal Processing, volume 2, pages II/125-II/128, 1994.

32. Young, S.: A Review of Large-Vocabulary Continuous-Speech Recognition. IEEE Signal Processing Magazine, 13:45-57, September 1996. 
33. Fine, S., Singer, Y., and Tishby, N.: The Hierarchical Hidden Markov Model: Analysis and Applications. Machine Learning, 32:41-62, 1998.

34. Oliver, N., Garg, A., and Horvitz, E.: Layered Representations for Learning and Inferring Office Activity from Multiple Sensory Channels. Computer Vision and Image Understanding, 96:163-180, 2004.

35. Ng, A. and Jordan, M.: On Discriminative vs. Generative classifiers: A comparison of logistic regression and naive Bayes. In Proceedings of the Neural Information Processing Systems Conference, volume 2, pages 841-848, 2002.

36. McCallum, A., Freitag, D., and Pereira, F.: Maximum Entropy Markov Models for Information Extraction and Segmentation. In Proceedings of the International Conference on Machine Learning, volume 1, pages 591-598, 2000.

37. Jaynes, E. T.: Information Theory and Statistical Mechanics. Physical Review, 106:620630, 1957.

38. Jaynes, E. T.: Information Theory and Statistical Mechanics II. Physical Review, 108:171190, 1957.

39. Lafferty, J., McCallum, A., and Pereira, F.: Conditional Random Fields: Probabilistic Models for Segmenting and Labeling Sequence Data. In Proceedings of the International Conference on Machine Learning, volume 1, pages 282-289, 2001.

40. Friedman, J., Bentley, J., and Finkel, R.: An Algorithm for Finding Best Matches in Logarithmic Expected Time. ACM Transactions on Mathematical Software, 3:209226, 1977.

41. de Berg, M., van Kreveld, M., Overmars, M., and Schwarzkopf, O.: Computational Geometry: Algorithms and Applications. Springer, 2000.

42. Silpa-Anan, C. and Hartley, R.: Optimised KD-Trees for Fast Image Descriptor Matching. In Proceedings of the IEEE Conference on Computer Vision and Pattern Recognition, 2008.

43. Nister, D. and Stewenius, H.: Scalable Recognition with a Vocabulary Tree. In Proceedings of the IEEE Conference on Computer Vision and Pattern Recognition, 2006. 
44. Muja, M. and Lowe, D.: Fast Approximate Nearest Neighbors with Automatic Algorithm Configuration. In VISAPP International Conference on Computer Vision Theory and Applications, 2009.

45. Kuhn, H. W.: The Hungarian Method for the Assignment Problem. Naval Research Logistics Quarterly, 2:83-97, 1955.

46. Center for Spoken Language Understanding: Speaker Recognition Version 1.1. http://www.ldc.upenn.edu/Catalog/CatalogEntry.jsp $?$ catalogId=LDC2006S26.

47. Cole, R., Noel, M., and Noel, V.: The CSLU Speaker Recognition Corpus. In International Conference on Spoken Language Processing, November 1998.

48. Lamel, L., Kassel, R., and Seneff, S.: Speech Database Development: Design and Analysis of the Acoustic-Phonetic Corpus. In Proceedings of the DARPA Speech Recognition Workshop, 1986.

49. Landgrebe, T. C. W. and Duin, R. P. W.: Approximating the Multiclass ROC by Pairwise Analysis. Pattern Recognition Letters, 28:1747-1758, 2007.

50. Rees, G. S., Wright, W., and Greenway, P.: ROC Method for the Evaluation of Multi-class Segmentation/Classification Algorithms with Infrared Imagery. In BMVC, pages 537-546, 2002.

51. Fawcett, T.: An Introduction to ROC Analysis. Pattern Recognition Letters, 27:861-874, 2006.

52. Bouvrie, J., Ezzat, T., and Poggio, T.: Localized Spectro-temporal Cepstral Analysis of Speech. In Proceedings of the International Conference on Acoustics, Speech and Signal Processing, pages 4733-4736, 2008. 


\section{VITA}

NAME

Simone Franzini

EDUCATION

B.S., Computer Science Engineering, Politecnico di Milano, Cremona, Italy, 2004

M.S., Computer Science Engineering, Politecnico di Milano, Milan, Italy, 2006

M.S., Computer Science, University of Illinois at Chicago, Chicago, Illinois, 2007

Ph.D., Computer Science, University of Illinois at Chicago, Chicago, Illinois, 2012

\section{TEACHING}

Department of Computer Science, University of Illinois at Chicago, Chicago, Illinois:

Computer Architecture I, S2007-S2008; Computer Architecture II, S2007-S2009;

C/C++ Programming for Engineers with Matlab, Su2009; Computer Graphics, F2008;

Advanced Computer Architecture, S2009; Introduction to Computing, F2009;

Data Structures and Discrete Mathematics I, S2010.

PROFESSIONAL MEMBERSHIPS

Institute of Electrical and Electronic Engineers (IEEE)

\section{PUBLICATIONS}

Franzini, S. and Ben-Arie, J.: Speech Recognition by Indexing and Sequencing, Proceedings of the International Conf. of Soft Computing and Pattern Recognition, pages 93-98, 2010.

Franzini, S. and Ben-Arie, J.: Speech Recognition by Indexing and Sequencing, International Journal of Comp. Inform. Syst. and Industr. Management Applications, 4:358-365, 2012. 